\title{
Stability of Shock Waves for the Broadwell Equations
}

\author{
Russel E. Caflisch ${ }^{1 \star}$ and Tai-Ping Liu ${ }^{2 \star \star}$ \\ ${ }^{1}$ Courant Institute of Mathematical Sciences, 251 Mercer Street, New York, NY 10012, USA \\ ${ }^{2}$ Mathematics Department, University of Maryland, College Park, MD 20742, USA
}

\begin{abstract}
For the Broadwell model of the nonlinear Boltzmann equation, there are shock profile solutions, i.e. smooth traveling waves that connect two equilibrium states. For weak shock waves, we prove asymptotic (in time) stability with respect to small perturbations of the initial data. Following the work of Liu [7] on shock wave stability for viscous conservation laws, the method consists of analyzing the solution as the sum of a shock wave, a diffusive wave, a linear hyperbolic wave and an error term. The diffusive and linear hyperbolic waves are approximate solutions of the fluid dynamic equations corresponding to the Broadwell model. The error term is estimated using a variation of the energy estimates of Kawashima and Matsumura [6] and the characteristic energy method of Liu [7].
\end{abstract}

\section{Introduction}

The Broadwell model for the nonlinear Boltzmann equation is

$$
\begin{aligned}
\left(\frac{\partial}{\partial t}+\frac{\partial}{\partial x}\right) f_{+} & =f_{0}^{2}-f_{+} f_{-}, \\
\frac{\partial}{\partial t} f_{0} & =-\frac{1}{2}\left(f_{0}^{2}-f_{+} f_{-}\right), \\
\left(\frac{\partial}{\partial t}-\frac{\partial}{\partial x}\right) f_{-} & =f_{0}^{2}-f_{+} f_{-},
\end{aligned}
$$

in which $f_{+}, f_{0}, f_{-}$represent the densities of particles moving with speeds $1,0,-1$ in the $x$ direction. The physical significance of $(1.1)$ is discussed in [2,3]. Global existence for solutions of the initial value problem for (1.1) is proved in [10] and the fluid dynamic limit for (1.1) is analyzed in [3].

* Research supported by the Office of Naval Research through grant N00014-81-0002 and by the National Science Foundation through grant NSF-MCS-83-01260

$\star \star$ Research supported by the National Science Foundation through grant DMS-84-01355 
Shock wave solutions of (1.1) are analyzed in $[2,4]$. They are traveling wave solutions $f=\left(f_{+}, f_{0}, f_{-}\right)(y=x-s t)$ solving

$$
\begin{aligned}
(1-s) \frac{\partial}{\partial y} f_{+} & =f_{0}^{2}-f_{+} f_{-}, \\
-s \frac{\partial}{\partial y} f_{0} & =-\frac{1}{2}\left(f_{0}^{2}-f_{+} f_{-}\right), \\
-(1+s) \frac{\partial}{\partial y} f_{-} & =f_{0}^{2}-f_{+} f_{-},
\end{aligned}
$$

for $-\infty<y<\infty$ with conditions

$$
\lim _{y \rightarrow-\infty} f(y)=\mathrm{g}^{-\infty}, \quad \lim _{y \rightarrow \infty} f(y)=\mathrm{g}^{\infty} .
$$

The limiting states $g^{\infty}=\left(g_{+}^{\infty}, g_{0}^{\infty}, g_{-}^{\infty}\right)$ and $g^{-\infty}=\left(g_{+}^{-\infty}, g_{0}^{-\infty}, g_{-}^{-\infty}\right)$ are equilibria satisfying

$$
g_{+}^{\infty} g_{-}^{\infty}=\left(g_{0}^{\infty}\right)^{2}, \quad g_{+}^{-\infty} g_{-}^{-\infty}=\left(g_{0}^{-\infty}\right)^{2} .
$$

Moreover $g^{\infty}, g^{-\infty}$, must be related by Rankine-Hugoniot and entropy conditions [4], i.e.

$$
\begin{aligned}
(1-s) g_{+}^{\infty}-4 s g_{0}^{\infty}-(1+s) g_{-}^{\infty} & =(1-s) g_{+}^{-\infty}-4 s g_{0}^{-\infty}-(1+s) g_{-}^{-\infty}, \\
(1-s) g_{+}^{\infty}+(1+s) g_{-}^{\infty} & =(1-s) g_{+}^{-\infty}+(1+s) g_{-}^{-\infty}, \\
s\left(g_{+}^{\infty}+4 g_{0}^{\infty}+g_{-}^{\infty}\right) & <s\left(g_{+}^{-\infty}+4 g_{0}^{-\infty}+g_{-}^{-\infty}\right) .
\end{aligned}
$$

Solutions of (1.2)-(1.3) can be written explicitly as hyperbolic tangents. Such a solution is called a weak shock wave if $g^{-\infty}$ and $g^{\infty}$ are close.

In this paper we prove asymptotic (in time) stability with respect to small perturbations in initial data for weak shock wave solutions of (1.1). The main result is the following theorem:

Theorem 1. There is a number $\delta>0$ for which the following is true: Let $f_{1}(x-s t)$ $=\left(f_{1+}, f_{10}, f_{1-}\right)(x-s t)$ solve $(1.2),(1.3)$ with

$$
\left|g_{+}^{\infty}-g_{+}^{-\infty}\right|^{2}+\left|g_{0}^{\infty}-g_{0}^{-\infty}\right|^{2}+\left|g_{-}^{\infty}-g_{-}^{-\infty}\right|^{2}<\delta^{2} .
$$

Let $f_{I}(x)=\left(f_{I+}, f_{I 0}, f_{I_{-}}\right)(x)$ be initial data that is uniformly bounded and satisfies

$$
\int\left(\left|f_{I}-f_{1}\right|+\left|f_{I}-f_{1}\right|^{2}+\left|f_{I x}-f_{1 x}\right|^{2}+\left|f_{I x x}-f_{1 x x}\right|^{2}\right) d x<\delta^{2} .
$$

Let $f(x, t)=\left(f_{+}, f_{0}, f_{-}\right)(x, t)$ solve (1.1) with $f(x, t=0)=f_{I}(x)$. Then there is a finite number $x_{0}$ such that for $f_{1}=f_{1}\left(x+x_{0}, t\right)$,

$$
\begin{aligned}
\sup _{t} & \int_{-\infty}^{\infty}\left|f-f_{1}\right|^{2}+ \\
+ & \left|f_{x}-f_{1 x}\right|^{2}+\left|f_{t}-f_{1 t}\right|^{2} d x \\
& \int_{-\infty}^{\infty}\left|f-f_{1}\right|^{2}+\left|f_{x}-f_{1 x}\right|^{2}+\left|f_{t}-f_{1 t}\right|^{2} d x d \tau \leqq c \delta^{2}, \\
& \lim _{t \rightarrow \infty} \int_{-\infty}^{\infty}\left|f_{x}-f_{1 x}\right|^{2}(x, t) d x=0 .
\end{aligned}
$$


Note. 1) The translation $x_{0}$ can be computed directly from the initial data, as in Eq. (3.4).

2) The existence of a unique solution $f(x, t)$ for all time is guaranteed by the global existence theory for the Broadwell equation [10]. For a survey of the theory, see [9].

3) Through the Sobolev inequality, (1.8) and (1.9) imply that $\lim \sup \left|f_{1}\left(x_{0}+x-s t\right)-f(x, t)\right|=0$.

$t \rightarrow \infty \quad x$

4) The solution produced in [10] may in general be exponentially growing. Our theorem shows that this is not the case for initial data close to a weak shock. An analogous result is proved in [1] for initial data with finite total mass. In that case the solution eventually decomposes into linear waves with characteristic speeds $\pm 1,0$ of (1.1). In the present case, the initial state has infinite mass and the results of [1] are not applicable. The asymptotic behavior of solutions is approximated by the fluid dynamic limit with characteristic speeds related to the sound speed.

The proof of this theorem is partly based on the fluid dynamic approximation, i.e. the Chapman-Enskog expansion, for the difference between the solution $f$ and the shock wave $f_{1}$. This approximation is valid for describing the nonlinear diffusion wave because the difference $f-f_{1}$ is small there. In the region of the shock, the difference $f-f_{1}$ consists mainly of a linear hyperbolic wave, which satisfies equations that are slightly different from the model Euler equations. Analogous stability results were proved by Liu for viscous conservation laws [7] and for the compressible Navier-Stokes equations [8]. Earlier results on stability for the Broadwell equations by Kawashima and Matsumura (abbreviated by KM) [6] and for viscous conservation laws by Goodman [5] and KM [6] are more restrictive in that they impose the constraint that the initial perturbation $f_{I}-f_{1}$ have no net (integral over $x$ ) mass or momentum, which precludes the diffusion wave.

Following Liu, the difference $f-f_{1}$ is decomposed into three parts: First there is a nonlinear diffusion wave $f_{2}$, which carries the net mass and momentum of $f-f_{1}$ and is an approximate solution of the model Navier-Stokes Eq. (2.14). The second part is a linear hyperbolic wave $f_{3}$ which corrects for the local mass and momentum errors in the diffusion wave but carries no net mass and momentum asymptotically in time. Selection of the correct linear hyperbolic Eq. (2.39), (2.40) for this wave is a crucial detail of this analysis. The third part is a remainder term $f_{4}$, which is estimated using a slight modification of the energy estimates of KM [6]. These energy estimates must be supplemented by estimates of the characteristic energy method [7] in regions where the diffusion wave is weakly expansive. Use of this method is the main difference between the present stability result and the result of KM [6]. In this paper the characteristic energy method is slightly simplified to use integration along the piecewise linear approximation of the characteristics. This was partly motivated by a suggestion from James Ralston.

The Broadwell equations are rewritten and the equations for diffusive waves and linear hyperbolic waves are derived in Sect. 2. The equation for the remainder and the error terms in that equation are described in Sect. 3. In Sect. 4 energy estimates are proved and in Sect. 5 the characteristic energy method is applied. The proof of Theorem 1 is summarized at the end of Sect. 5. 


\section{The Broadwell Equation and the Model Fluid Dynamic Equations}

\section{A. The Broadwell Equation and Shock Profile}

Rewrite the Broadwell Eq. (1.1) as

$$
\begin{gathered}
\varrho_{t}+m_{x}=0, \quad m_{t}+z_{x}=0, \\
z_{t}+m_{x}=\frac{1}{8}\left\{(\varrho-z)^{2}-4\left(z^{2}-m^{2}\right)\right\} \equiv Q(f, f),
\end{gathered}
$$

in which $\varrho=f_{+}+4 f_{0}+f_{-}$is the local mass density, $m=f_{+}-f_{-}$is the local momentum and $z=f_{+}+f_{-}$. For convenience denote now $f=(\varrho, m, z)$ and define the quadratic form $Q$ to be the right-hand side of (2.1).

The shock wave solutions of (2.1) [corresponding to solutions of (1.2), (1.3)] are traveling waves $(\varrho, m, z)(x, t)=\left(\varrho_{1}, m_{1}, z_{1}\right)(\xi=x$-st $)$ solving

$$
\begin{gathered}
-s \varrho_{1_{\xi}}+m_{1_{\xi}}=0, \quad-s m_{1_{\xi}}+z_{1_{\xi}}=0, \\
-s z_{1_{\xi}}+m_{1_{\xi}}=\frac{1}{8}\left\{\left(\varrho_{1}-z_{1}\right)^{2}-4\left(z_{1}^{2}-m_{1}^{2}\right)\right\}
\end{gathered}
$$

with limiting values

$$
\lim _{\xi \rightarrow \infty}\left(\varrho_{1}, m_{1}, z_{1}\right)=\left(\varrho_{1}^{\infty}, m_{1}^{\infty}, z_{1}^{\infty}\right), \quad \lim _{\xi \rightarrow-\infty}\left(\varrho_{1}, m_{1}, z_{1}\right)=\left(\varrho_{1}^{-\infty}, m_{1}^{-\infty}, z_{1}^{-\infty}\right)
$$

which are in equilibrium,

$$
\left(\varrho_{1}^{\infty}-z_{1}^{\infty}\right)^{2}=4\left(z_{1}^{\infty}\right)^{2}-4\left(m_{1}^{\infty}\right)^{2}, \quad\left(\varrho_{1}^{-\infty}-z_{1}^{-\infty}\right)^{2}=4\left(z_{1}^{-\infty}\right)^{2}-4\left(m_{1}^{-\infty}\right)^{2},
$$

satisfy Rankine-Hugoniot conditions

$$
-s \varrho_{1}^{-\infty}+m_{1}^{-\infty}=-s \varrho_{1}^{\infty}+m_{1}^{\infty}, \quad-s m_{1}^{-\infty}+z_{1}^{-\infty}=-s m_{1}^{\infty}+z_{1}^{\infty},
$$

and satisfy an entropy condition

$$
S Q_{1}^{\infty}<S \varrho_{1}^{-\infty} .
$$

For any limiting states satisfying (2.4), (2.5), (2.6) a unique solution of (2.2), (2.3) is easily constructed [4].

For the sake of definiteness we assume that $\left(\varrho_{1}, m_{1}, z_{1}\right)$ is a forward shock wave, which here just means that the shock speed $s$ is positive. The speed $s$ also satisfies the stability condition [4]

$$
\lambda_{2}\left(\varrho_{1}^{-\infty}, m_{1}^{-\infty}\right)>s>\lambda_{2}\left(\varrho_{1}^{\infty}, m_{1}^{\infty}\right)
$$

in which $\lambda_{1}, \lambda_{2}$, satisfying $\lambda_{1}<0<\lambda_{2}$, are the characteristic speeds of the model Euler equations [cf. (2.13)] described in the next section.

The explicit form of the shock wave is given by

$$
\left(\begin{array}{l}
\varrho_{1} \\
m_{1} \\
z_{1}
\end{array}\right)=-\frac{1}{2}\left(\begin{array}{c}
Q^{-\infty}-Q^{\infty} \\
m^{-\infty}-m^{\infty} \\
z^{-\infty}-z^{\infty}
\end{array}\right) \tanh (\kappa(x+\bar{x}-s t))+\frac{1}{2}\left(\begin{array}{c}
\varrho^{-\infty}+Q^{\infty} \\
m^{-\infty}+m^{\infty} \\
z^{-\infty}+z^{\infty}
\end{array}\right)
$$

in which $\bar{x}$ is an arbitrary constant, with $\kappa=\left(1+3 s^{2}\right)(16 s)^{-1}\left(\varrho^{-\infty}-\varrho^{\infty}\right)[4]$. 


\section{B. Model Fluid Equations}

By assuming that $f=(\varrho, m, z)$ is a local Maxwellian satisfying $Q(f, f)=0$ at every $(x, t)$ and by dropping the third equation of (2.1), the following model Euler equations are obtained:

$$
\varrho_{t}+m_{x}=0, \quad m_{t}+\hat{z}(\varrho, m)_{x}=0,
$$

in which

$$
\hat{z}(\varrho, m)=\varrho F(u)
$$

with

$$
u=m / \varrho, \quad F(u)=\left(2 \sqrt{1+3 u^{2}}-1\right) / 3 .
$$

The Euler Eqs. (2.9) can be rewritten as

$$
\left(\begin{array}{c}
\varrho \\
m
\end{array}\right)_{t}+\left(\begin{array}{cc}
0 & 1 \\
a / b & m / b
\end{array}\right)\left(\begin{array}{c}
\varrho \\
m
\end{array}\right)_{x}=0
$$

with

$$
a=(\varrho-\hat{z}) / 4, \quad b=(\varrho+3 \hat{z}) / 4 .
$$

The characteristic speeds of $\left(2.9^{\prime}\right)$ are

$$
\begin{aligned}
& \lambda_{1}=\frac{m-\sqrt{m^{2}+4 a b}}{2 b}=-\frac{2 a}{m+\sqrt{m^{2}+4 a b}}, \\
& \lambda_{2}=\frac{m+\sqrt{m^{2}+4 a b}}{2 b}=\frac{2 a}{-m+\sqrt{m^{2}+4 a b}} .
\end{aligned}
$$

If the components $f_{+}, f_{0}, f_{-}$of the solution $f$ are initially nonnegative, they remain nonnegative [1]. For such a solution $|u| \leqq 1$ (i.e., the average velocity is no larger than the molecular speed) and $a>0, b>0$. Thus $\lambda_{1}<0<\lambda_{2}$.

A better approximation of (2.1) is given by the model Navier-Stokes equations, which are obtained from (2.1) through the Chapman-Enskog expansion [3] as

$$
\varrho_{t}+m_{x}=0, \quad m_{t}+\tilde{z}(\varrho, m)_{x}=0,
$$

in which

$$
\tilde{z}(\varrho, m)=\hat{z}(\varrho, m)-v(u) u_{x} .
$$

The viscosity function $v$ is

$$
v(u)=2(1-F(u))\left(1+3 u^{2}\right)^{-3 / 2} .
$$

The Euler equations (2.9) have discontinuous shock wave solutions, while the Navier-Stokes equations (2.14) have smooth shock profile solutions, which approximate the shock wave solutions of the Broadwell equation (2.1) if the shocks are weak.

Just as for the real Navier-Stokes equations [8], the shock waves are compressive; that is the associated characteristic speed $\lambda_{2}$ (for forward shocks) 
decreases across the shock. As a consequence, $\lambda_{1}, \lambda_{2}, a$ and $b$ defined by (2.13) are strictly monotone across a viscous shock wave for (2.14). For a weak shock wave of (2.1) KM [6] showed that

$$
\frac{\partial}{\partial \xi}\left(\lambda_{1}\right)<0, \quad \frac{\partial}{\partial \xi}\left(\lambda_{2}\right)<0
$$

in which $\lambda_{1}, \lambda_{2}$ are evaluated at the shock wave $f=f_{1}$. Also since the shock wave is forward (with approximate speed $\lambda_{2}$ ), and since the shock wave decays exponentially away from its center, it satisfies

$$
\begin{gathered}
\left|\partial_{t} f_{1}+\lambda_{2} \partial_{x} f_{1}\right|<c \delta\left|\lambda_{2 x}\right| \\
\left|\chi_{+}\left(f_{1}-f_{1}(\infty)\right)\right|+\left|\chi_{-}\left(f_{1}-f_{1}(-\infty)\right)\right|+\left|\partial_{x} f_{1}\right|+\left|\partial_{t} f_{1}\right|<c\left|\lambda_{2 x}\right|<c \delta e^{-\gamma|x-s t|},
\end{gathered}
$$

in which $\chi_{+}=1$ for $x>$ st, $\chi_{+}=0$ for $x<s t, \chi_{-}=1-\chi_{+}$, and $\gamma$ is some constant.

\section{C. Diffusion Waves}

Liu [7] showed that, for a viscous conservation law such as (2.14), a small perturbation of a constant evolves approximately as a diffusion wave. For the forward shock wave solution $\left(\varrho_{1}, m_{1}, z_{1}\right)$ of $(2.2)$, the diffusion wave $\left(\varrho_{2}, m_{2}\right)$ moves backward, and so it is a perturbation of the limiting state $\left(\varrho_{1}^{-\infty}, m_{1}^{-\infty}, z_{1}^{-\infty}\right)$. As in [7], the diffusion wave solves

$$
\begin{aligned}
& \varrho_{2 t}+m_{2 x}=e_{1}, \\
& m_{2 t}+\tilde{z}_{2 x}=e_{2},
\end{aligned}
$$

in which

$$
\tilde{z}_{2}=\tilde{z}\left(\varrho_{2}+\varrho_{1}^{-\infty}, m_{2}+m_{1}^{-\infty}\right)-z_{1}^{-\infty} .
$$

From now on we write $f_{2} \equiv\left(\varrho_{2}, m_{2}, \tilde{z}_{2}\right)$. The error terms $e_{1}, e_{2}$ are chosen as in Sect. 3 of [7] so that (2.19), (2.20) is equivalent to Burger's equation.

To be precise, $f_{2}$ is uniquely determined by the following properties:

(i) $\left(\varrho_{2}+\varrho_{1}^{-\infty}, m_{2}+m_{1}^{-\infty}\right)$ lies on the integral curve of the right eigenvector $r_{1}$ through $\left(\varrho_{1}^{-\infty}, m_{1}^{-\infty}\right)$,

(ii) $\tilde{\lambda}_{1} \equiv \lambda_{1}\left(\varrho_{2}+\varrho_{1}^{-\infty}, m_{2}+m_{1}^{-\infty}\right)-\lambda_{1}^{-\infty}$ is a self-similar solution of the Burgers equation, i.e.

$$
\begin{aligned}
& \tilde{\lambda}_{1}(x, t)= {\left[(\exp (\kappa \delta / 2 \sqrt{\alpha})-1)(\mathrm{t}+1)^{-1 / 2} \exp \left(-y^{2}\right)\right] } \\
& \times {\left[2 \sqrt{\pi / \alpha}+(\exp (\kappa \delta / 2 \sqrt{\alpha})-1) \int_{y}^{\infty} \alpha^{-1 / 2} e^{-\xi^{2}} d \xi\right]^{-1}, } \\
& y \equiv\left(x-(t+1) \lambda_{1}^{-\infty}\right) / 2 \sqrt{\alpha(t+1)}
\end{aligned}
$$

The quantities $r_{1}$ and $\alpha \equiv \alpha_{+}\left(\varrho_{1}^{-\infty}, m_{1}^{-\infty}\right)$ are defined in (2.26), (2.28) below. $\kappa$ has value 1 or -1 for diffusion wave with positive or negative mass. Here $\kappa \delta$ replaces $\delta$ in [7], since we now take $\delta>0$.

For the construction of the diffusion wave from Burger's equation, the viscosity matrix of (2.14) must be expressed in the basis of left and right eigenvectors of the 
convection matrix of $\left(2.9^{\prime}\right)$. The viscosity matrix is

$$
V=\left(\begin{array}{cc}
0 & 0 \\
-a m b^{-3} & a \varrho b^{-3}
\end{array}\right) .
$$

The left eigenvectors $l_{ \pm}$and right eigenvectors $r_{ \pm}$of the convection matrix are

$$
\begin{gathered}
l_{1}=\left(\left(-m-\sqrt{m^{2}+4 a b}\right) / 2 b, 1\right), \quad l_{2}=\left(\left(-m+\sqrt{m^{2}+4 a b}\right) / 2 b, 1\right), \\
r_{1}=\left(-2 b / \sqrt{m^{2}+4 a b}, 1-m / \sqrt{m^{2}+4 a b}\right)^{\dagger}, \\
r_{2}=\left(2 b / \sqrt{m^{2}+4 a b}, 1+m / \sqrt{\left.m^{2}+4 a b\right)^{\dagger}} .\right.
\end{gathered}
$$

In the coordinate system $r_{1}, r_{2}$, the viscosity matrix becomes

$$
\left(\begin{array}{l}
l_{1} \\
l_{2}
\end{array}\right) V\left(r_{1} r_{2}\right)=\left(\begin{array}{ll}
\alpha_{+} & \alpha_{-} \\
\alpha_{+} & a_{-}
\end{array}\right)
$$

with

$$
\alpha_{ \pm}=\frac{ \pm m(2 b-\varrho)+\varrho \sqrt{m^{2}+4 a b}}{\sqrt{m^{2}+4 a b}} \cdot \frac{a}{b^{3}} .
$$

Using the facts that $a>0, b>0$ and $|F(u)|<1$, it is easily shown that the diagonal elements of (2.26) are positive, i.e. $\alpha_{ \pm}>0$, which is needed for the construction of the diffusion wave $f_{2}$.

Define

$$
\Omega_{1} \equiv\{(x, t), t \geqq 0, x \leqq 0\}, \quad \Omega_{2} \equiv\{(x, t), t \geqq 0, x \geqq 0\} .
$$

It follows as in Sect. 5 of [7] that the set $\Omega_{1}$ can be divided into two parts $\Omega_{+}$and $\Omega_{-}$such that the characteristics for $\left(\varrho_{2}, m_{2}\right)$ are compressive in $\Omega_{-}$and weakly expansive in $\Omega_{+}$, i.e.

$$
\frac{\partial \lambda_{1}\left(\varrho_{2}, m_{2}\right)}{\partial x}<0 \quad \text { in } \quad \Omega_{-}, \quad \frac{\partial \lambda_{1}\left(\varrho_{2}, m_{2}\right)}{\partial x}>0 \text { in } \Omega_{+} .
$$

Moreover $\Omega_{ \pm}$are characterized by (with $y$ defined in (2.23))

$$
\begin{array}{lll}
\Omega_{+}\left(\Omega_{-}\right) \equiv\left\{(x, t) \in \Omega_{1}: y \geqq y_{1}\left(y \leqq y_{1}\right)\right\} & \text { if } \quad \kappa=1, \\
\Omega_{+}\left(\Omega_{-}\right) \equiv\left\{(x, t) \in \Omega_{1}: y \leqq y_{1}\left(y \geqq y_{1}\right)\right\} & \text { if } \quad \kappa=-1,
\end{array}
$$

for some constant $y_{1}$, as shown in Fig. 1. Define also $\Omega_{ \pm}(t)=\Omega_{ \pm} \cap\{(x, \tau): 0<\tau<t\}$, $\Omega_{i}(t)=\Omega_{i} \cap\{(x, \tau): 0 \leqq \tau<t\}$ for $i=1,2$.

The nonlinear diffusion wave satisfies the following bounds (cf. (3.8) in [7]):

$$
\begin{aligned}
\left|f_{2}\right| & \simeq\left|\varrho_{2}\right|+\left|m_{2}\right|+\left|z_{2}\right|+\left|u_{2}\right| \leqq c \delta(t+1)^{-1 / 2} e^{-y^{2}}, \\
\left|f_{2 x}\right| & \leqq c \delta(t+1)^{-1}(|y|+1) e^{-y^{2}} \\
\left|f_{2 t}\right| & \leqq c \delta(t+1)^{-3 / 2}\left(|y|^{2}+1\right) e^{-y^{2}} \\
\left|f_{2 x t}\right| & \leqq c \delta(t+1)^{-2}\left(|y|^{3}+1\right) e^{-y^{2}} \\
\left|f_{2 x x}\right| & \leqq c \delta(t+1)^{-3 / 2}\left(|y|^{2}+1\right) e^{-y^{2}}
\end{aligned}
$$



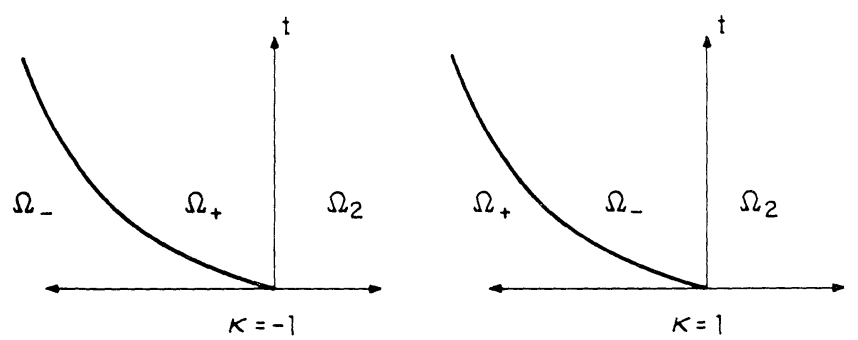

Fig. 1. Regions $\Omega_{+}, \Omega_{-}, \Omega_{2}$ in $(x, t)$ for the two cases $\kappa=1$ and $\kappa=-1$

in which $y$ is defined by (2.23). Moreover since $f_{2}$ is determined through its eigenvalue $\lambda_{1}$, it follows that

$$
\begin{gathered}
\left|\partial_{x} f_{2}\right|<c\left|\partial_{x} \lambda_{1}\right|, \quad\left|\partial_{t} f_{2}\right|<c\left|\partial_{t} \lambda_{1}\right|, \\
\left(\partial_{t}+\lambda_{1} \partial_{x}\right) f_{2}=O(1) \partial_{x x} \lambda_{1} .
\end{gathered}
$$

Using (6.12), (6.13), (3.5) of [7], the error terms $e_{1}, e_{2}$ satisfy the bound

$$
\left|e_{i}\right| \leqq c \delta(1+t)^{-3 / 2}\left(|y|^{2}+1\right) e^{-y^{2}}
$$

\section{D. Linear Hyperbolic Wave}

A linear hyperbolic wave $\left(\varrho_{3}, m_{3}, \tilde{z}_{3}\left(\varrho_{3}, m_{3}\right)\right)$ is needed as in [7] to compensate for the errors $e_{1}, e_{2}$ in (2.19), (2.20). The equations for $\varrho_{3}, m_{3}$ are

$$
\begin{gathered}
\varrho_{3 t}+m_{3 x}=-e_{1}, \quad m_{3 t}+\tilde{z}_{3}\left(\varrho_{3}, m_{3}\right)_{x}=-e_{2}, \\
\left(\varrho_{3}, m_{3}\right)(x, t) \rightarrow 0 \text { as } t \rightarrow \infty,
\end{gathered}
$$

in which

$$
\tilde{z}_{3}\left(\varrho_{3}, m_{3}\right)=\frac{A}{B} \varrho_{3}+\frac{M}{B} m_{3},
$$

with $A, B, M$ depending on $\left(\varrho_{1}, m_{1}, z_{1}\right)$ and $\left.\left(\varrho_{2}, m_{2}\right)\right)$ as

$$
\begin{gathered}
A=\frac{1}{4}\left\{\left(\varrho_{1}+\varrho_{2}\right)-\left(z_{1}+\tilde{z}_{2}\right)\right\}, \quad B=\frac{1}{4}\left\{\left(\varrho_{1}+\varrho_{2}\right)+3\left(z_{1}+\tilde{z}_{2}\right)\right\}, \\
M=m_{1}+m_{2} .
\end{gathered}
$$

The form of $\tilde{z}_{3}$ is chosen so that $f_{2}+f_{3}$, together with the shock wave $f_{1}$, forms an accurate approximate solution [cf. (3.11)]. Clearly from (2.19), (2.20), and (2.39),

$$
\frac{d}{d t} \int_{-\infty}^{\infty}\left(\varrho_{2}+\varrho_{3}\right)(x, t) d x=0, \quad \frac{d}{d t} \int_{-\infty}^{\infty}\left(m_{2}+m_{3}\right)(x, t) d x=0
$$

for $t \geqq 0$. Thus if the net mass and momentum of the initial perturbation is contained in $\left(\varrho_{2}, m_{2}\right)+\left(\varrho_{3}, m_{3}\right)$, it will remain there for all time. Moreover the construction in Sect. 3 of $\left(\varrho_{2}, m_{2}\right)$ and $\left(\varrho_{3}, m_{3}\right)$ guarantees that

$$
\int_{-\infty}^{\infty}\left(\begin{array}{c}
\varrho_{2}+\varrho_{3} \\
m_{2}+m_{3}
\end{array}\right)(x, t) d x=\bar{c} \delta r_{1}^{-\infty}
$$


in which $\bar{c}$ is a constant and $r_{1}^{-\infty}=r_{1}\left(\varrho_{1}^{-\infty}, m_{1}^{-\infty}\right)$ is the right eigenvector defined in (2.26).

As shown in [7] the error terms $\left(e_{1}, e_{2}\right)$ have a good form and decay rate. The initial data is appropriately chosen as in [7] so that $f_{3} \rightarrow 0$ as $t \rightarrow \infty$. It follows that $f_{3} \equiv\left(\varrho_{3}, m_{3}, \tilde{z}_{3}\right)$, with $\varrho_{3}, m_{3}$ solving (2.39), has the following decay properties (cf. Theorems $7.5,7.6$ of [7])

$$
\begin{gathered}
\left|f_{3}(x, t)\right| \leqq c \delta\left\{\left[1+t+t y^{2}\right]^{-1}+\left[1+t+t^{1 / 2}|y|\right]^{-3 / 2}\right\} \\
\left|f_{3 t}\right|+\left|f_{3 x}(x, t)\right|+\left|f_{3 x x}(x, t)\right| \\
\leqq c \delta\left\{\left[1+t+t y^{2}\right]^{-3 / 2}+\left[1+t+t^{1 / 2}|y|\right]^{-2}+|t+1|^{-3 / 2}\left(\left|f_{1 x}\right|+\left|f_{2 x}\right|\right)\right\} .
\end{gathered}
$$

\section{Wave Decomposition of the Solution}

The equations are reformulated here in terms of $\varrho, m, z$. Consider initial data $f_{I}=\left(\varrho_{I}, m_{I}, z_{I}\right)(x)$ that is a perturbation of a given forward shock wave solution $\left(\varrho_{1}, m_{1}, z_{1}\right)(\xi)$, i.e.

$$
\left(\varrho_{I}, m_{I}, z_{I}\right)(x)=\left(\varrho_{1}, m_{1}, z_{1}\right)(x)+\left(\varrho^{\prime}, m^{\prime}, z^{\prime}\right)(x, 0)
$$

with $\left(\varrho^{\prime}, m^{\prime}, z^{\prime}\right)(\cdot, 0) \in L^{1}(x)$ and $\lim _{x \rightarrow \pm \infty}\left(\varrho^{\prime}, m^{\prime}, z^{\prime}\right)(x, 0)=0$. The solution $(\varrho, m, z)(x, t)$ of the Broadwell equation with this initial data is written as

$$
(\varrho, m, z)(x, t)=\left(\varrho_{1}, m_{1}, z_{1}\right)(x-s t)+\left(\varrho^{\prime}, m^{\prime}, z^{\prime}\right)(x, t) .
$$

From (2.1) the solution has two time-invariant quantities, mass and momentum, i.e.

$$
\int_{-\infty}^{\infty}\left(\begin{array}{c}
\varrho^{\prime} \\
m^{\prime}
\end{array}\right)(x, t) d x=\int_{-\infty}^{\infty}\left(\begin{array}{c}
\varrho^{\prime} \\
m^{\prime}
\end{array}\right)(x, 0) d x
$$

for all $t>0$. For a weak forward shock wave the jump $\left[\varrho_{1}, m_{1}\right]$ $=\left(\varrho_{1}^{+\infty}, m_{1}^{+\infty}\right)-\left(\varrho_{1}^{-\infty}, m_{1}^{-\infty}\right)$ is nearly equal to the right eigenvector $r_{2}\left(\varrho_{1}^{-\infty}, m_{1}^{-\infty}\right)$ defined in (2.26). It follows that $\left[\varrho_{1}, m_{1}\right]$ and $r_{1}\left(\varrho_{1}^{-\infty}, m_{1}^{-\infty}\right)^{\dagger}$ are linearly independent and that the net perturbed mass and momentum can be written as a linear combination of them, i.e. for some constants $\bar{c}$ and $x_{0}$,

Note that

$$
\int_{-\infty}^{\infty}\left(\varrho^{\prime}, m^{\prime}\right)(x, t) d x=\bar{c} \delta r_{1}\left(\varrho_{1}^{-\infty}, m_{1}^{-\infty}\right)^{\dagger}+x_{0}\left[\varrho_{1}, m_{1}\right] .
$$

$$
\int_{-\infty}^{\infty}\left\{\left(\varrho_{1}, m_{1}\right)\left(x+x_{0}-s t\right)-\left(\varrho_{1}, m_{1}\right)(x-s t)\right\} d x=x_{0}\left[\varrho_{1}, m_{1}\right] .
$$

Thus for convenience we can take $x_{0}$ in (3.4) to be zero after replacing $\left(\varrho_{1}, m_{1}, z_{1}\right)(x-s t)$ in $(3.1),(3.2)$ by $\left(\varrho_{1}, m_{1}, z_{1}\right)\left(x+x_{0}-s t\right)$.

With $x_{0}$ set to zero this way and $\bar{c}$ defined by (3.4), the solution $(\varrho, m, z)$ is decomposed into the shock wave, diffusion wave, linear hyperbolic wave and remainder, i.e.

$$
\begin{aligned}
(\varrho, m, z)(x, t)= & \left(\varrho_{1}, m_{1}, z_{1}\right)(x-s t)+\sum_{i=2}^{3}\left(\varrho_{i}, m_{i}, \tilde{z}_{i}\left(\varrho_{v}, m_{i}\right)\right)(x, t) \\
& +\left(\varrho_{*}, m_{*}, z_{*}\right)(x, t)
\end{aligned}
$$


The diffusion wave $\left(\varrho_{2}, m_{2}, \tilde{z}_{2}\right)$ satisfies (2.19), (2.20) the linear hyperbolic wave $\left(\varrho_{3}, m_{3}, \tilde{z}_{3}\right)$ satisfies $(2.39)$, and together they satisfy $(2.43)$.

Combine the equations for $\left(\varrho_{i}, m_{i}, z_{i}\right)(i=1,2,3)$ together with Eq. (2.1) for $(\varrho, m, z)$ to obtain the following equation for $\left(\varrho_{*}, m_{*}, z_{*}\right)$ :

$$
\begin{gathered}
\varrho_{* t}+m_{* x}=0, \quad m_{* t}+z_{* x}=0 \\
z_{* t}+m_{* x}=G \equiv Q(f, f)-\left(z_{1}+\tilde{z}_{2}+\tilde{z}_{3}\right)_{t}-\left(m_{1}+m_{2}+m_{3}\right)_{x} .
\end{gathered}
$$

The initial data for $f_{*}=\left(\varrho_{*}, m_{*}, z_{*}\right)$ is defined through (3.6) since the initial values of $\left(\varrho_{i}, m_{i}, z_{i}\right) i=1,2,3$ are already chosen. Because of $(1.7) f_{*}$ satisfies

$$
\int\left|f_{*}\right|+\left|f_{*}\right|^{2}+\left|f_{* x}\right|^{2}+\left|f_{* x x}\right|^{2} d x \leqq \delta^{2}
$$

for $t=0$. Because of (2.43) and (3.4) with $x_{0}=0, f_{*}$ has no net mass or momentum, i.e.

$$
\int_{-\infty}^{\infty}\left(\varrho_{*}, m_{*}\right)(x, t) d x=0
$$

We wish to show that $(\varrho, m, z) \rightarrow\left(\varrho_{1}, m_{1}, z_{1}\right)$ as $t \rightarrow \infty$. Since $\left(\varrho_{i}, m_{i}, z_{i}\right) \rightarrow 0$ for $i=2,3$, we need only show that $\left(\varrho_{*}, m_{*}, z_{*}\right) \rightarrow 0$.

First we rearrange the right-hand side $G$ in (3.7). Decompose $G$ as

$$
G=H+2 Q\left(f_{1}+f_{2}+f_{3}, f_{*}\right)+Q\left(f_{*}, f_{*}\right),
$$

in which $f_{i}=\left(\varrho_{i}, m_{i}, z_{i}\right), f_{*}=\left(\varrho_{*}, m_{*}, z_{*}\right)$. The parts of $G$ containing $f_{*}$ are

$$
\begin{gathered}
Q\left(f_{1}+f_{2}, f_{*}\right)=\frac{1}{2}\left(A \varrho_{*}+M m_{*}-B z_{*}\right), \\
Q\left(f_{3}, f_{*}\right)=\frac{1}{8}\left(\varrho_{3}-\tilde{z}_{3}\right) \varrho_{*}+\frac{1}{2} m_{3} m_{*}-\frac{1}{8}\left(\varrho_{3}+3 \tilde{z}_{3}\right) z_{*}, \\
Q\left(f_{*}, f_{*}\right)=\Gamma\left(\varrho_{*}, m_{*}, z_{*}\right) \equiv \frac{1}{8}\left\{\left(\varrho_{*}-z_{*}\right)^{2}-4\left(z_{*}^{2}-m_{*}^{2}\right)\right\},
\end{gathered}
$$

in which $A, B, M$ are defined in (2.41).

For the linear hyperbolic wave, $z_{3}=\tilde{z}_{3}\left(\varrho_{3}, m_{3}\right)$ was chosen in $(2.40)$ so that

$$
Q\left(f_{1}+f_{2}, f_{3}\right)=0 \text {. }
$$

Also use the equation for $f_{1}$ to find that the inhomogeneous part of $G$ is $H=H_{1}+H_{2}+H_{3}$, in which

$$
\begin{gathered}
H_{1}=Q\left(f_{1}^{-\infty}+f_{2}, f_{1}^{-\infty}+f_{2}\right)-\left(z_{1}^{-\infty}+\tilde{z}_{2}\right)_{t}-\left(m_{1}^{-\infty}+m_{2}\right)_{x}, \\
H_{2}=2 Q\left(f_{1}-f_{1}^{-\infty}, f_{2}\right), \\
H_{3}=Q\left(f_{3}, f_{3}\right)-\tilde{z}_{3 t}-m_{3 x},
\end{gathered}
$$

since $Q\left(f_{1}^{-\infty}, f_{1}^{-\infty}\right)=\left(z_{1}^{-\infty}\right)_{t}=\left(m_{1}^{-\infty}\right)_{x}=0$. The term $H_{1}$ is the error in the third equation of (2.1) in which $f$ is replaced by a solution $f_{2}+f_{1}^{-\infty}$ of the ChapmanEnskog expansion (i.e. a solution of the model Navier-Stokes equations). A straightforward calculation in Appendix A shows that

$$
H_{1}=-\left(F_{2}-u_{2} F_{2}^{\prime}\right) e_{1}-F_{2}^{\prime} e_{2}-F_{2}^{\prime}\left(v_{2} u_{2 x}\right)_{x}+\left(v_{2} u_{2 x}\right)_{t}-\frac{3}{8}\left(v_{2} u_{2 x}\right)^{2},
$$

in which $F_{2}=F\left(u_{1}^{-\infty}+u_{2}\right), v_{2}=v\left(u_{1}^{-\infty}+u_{2}\right)$. 
Because of (3.8) it is natural to introduce

$$
(\phi, \psi)(x, t)=\int_{-\infty}^{x}\left(\varrho_{*}, m_{*}\right)(y, t) d y
$$

with $(\phi, \psi)(x= \pm \infty, t)=(0,0)$. Integrate the first two equations of (3.7) and eliminate $z_{*}$ to rewrite this system as

$$
\begin{aligned}
& \phi_{t}+\psi_{x}=0 \\
& \psi_{t}+(A / B) \phi_{x}+(M / B) \psi_{x}= B^{-1}\left(\psi_{x x}-\psi_{t t}-\Gamma\left(\phi_{x}, \psi_{x},-\psi_{t}\right)\right. \\
&\left.-H-K\left(\phi_{x}, \psi_{x}, \psi_{t}\right)\right)
\end{aligned}
$$

in which $H=H_{1}+H_{2}+H_{3}$ is defined in (3.12)-(3.14), $\Gamma$ is defined in (3.10) and

$$
K\left(\phi_{x}, \psi_{x}, \psi_{t}\right)=Q\left(f_{3}, f_{*}\right)=\frac{1}{8}\left(\varrho_{3}-z_{3}\right) \phi_{x}+\frac{1}{2} m_{3} \psi_{x}-\frac{1}{8}\left(\varrho_{3}+3 z_{3}\right) \psi_{t} .
$$

\section{Energy Estimates}

In this section energy estimates are derived for the system (3.17), (3.18). These estimates are the same as the estimates of Kawashima and Matsumura [6], except for small changes caused by the inclusion of $f_{2}$ in $A, B, M$ and the terms $H$ and $K$. However, because of these small changes, the energy estimates do not close: on the weakly expansive region $\Omega_{+}$of the diffusive wave $f_{2}$, a second set of characteristic energy estimates are needed and are derived in the next section.

First change variables from $(x, t)$ to $(\xi, t)$ with $\xi=x-s t$ and $s$ the shock speed. Note that $f_{2}$ is not a traveling wave with speed $s$; thus in the present problem $A, B$, $M$ depend on $t$ as well as $\xi$, in contrast to [6]. Rewrite (3.17), (3.18) as

$$
\begin{gathered}
L_{1}(\phi, \psi)=0, \\
L_{2}(\phi, \psi)=-\Gamma_{1},
\end{gathered}
$$

in which

$$
\begin{gathered}
L_{1}(\phi, \psi) \equiv \phi_{t}-s \phi_{\xi}+\psi_{\xi}, \\
L_{2}(\phi, \psi) \equiv\left(\psi_{t}-s \psi_{\xi}\right)_{t}-s\left(\psi_{t}-s \psi_{\xi}\right)_{\xi}-\psi_{\xi \xi}+A \phi_{\xi}+(M-s B) \psi_{\xi}+B \psi_{t}, \\
\Gamma_{1} \equiv \Gamma\left(\phi_{\xi}, \psi_{\xi},-\left(\psi_{t}-s \psi_{\xi}\right)\right)+K\left(\phi_{\xi}, \psi_{\xi}, \psi_{t}-s \psi_{\xi}\right)+H .
\end{gathered}
$$

The initial data for (4.1), (4.2) is

$$
(\phi, \psi)(\xi, 0)=\left(\phi_{0}, \psi_{0}\right)(\xi), \quad \psi_{t}(\xi, 0)=s \psi_{0 \xi}-z_{*}(\xi) .
$$

Define the following three norms for $\psi, \phi$ :

$$
\begin{gathered}
N_{1}(t)=\sup _{0 \leqq \tau \leqq t}\left(\|\phi, \psi\|_{2}(\tau)+\left\|\psi_{t}\right\|_{1}(\tau)\right), \\
N_{2}(t)=\left\{\int_{0}^{t}\left\|\phi_{\xi}, \psi_{\xi}, \psi_{t}\right\|_{1}^{2} d \tau\right\}^{1 / 2}, \\
N_{3}(t)=\left\{\int_{0}^{t} \int_{-\infty}^{\infty}\left(\left|\lambda_{1 \xi}\right|+\left|\lambda_{2 \xi}\right|\right)\left(\phi^{2}+\psi^{2}\right) d \xi d \tau\right\}^{1 / 2},
\end{gathered}
$$


in which \|\|$=\|\|_{0}$ and \|\|$_{k}$ is the $k^{\text {th }}$ Sobolev norm in space, e.g.

$$
\|\phi\|_{k}=\left(\int_{-\infty}^{\infty} \phi^{2}+\ldots+\left(\partial_{\xi}^{k} \phi\right)^{2} d \xi\right)^{1 / 2} .
$$

In these definitions we could replace $\xi$ by $x$ everywhere (without any changes) and correspondingly change $\left.\frac{\partial}{\partial t}\right|_{\xi}$ to $\left.\frac{\partial}{\partial t}\right|_{x}$.

Before proving the energy estimates, we derive two lemmas concerning the shock wave $f_{1}$, diffusion wave $f_{2}$ and linear hyperbolic wave $f_{3}$. Define $A_{ \pm}=\lim _{\xi \rightarrow \pm \infty} A(\xi, t), B_{ \pm}=\lim _{\xi \rightarrow \pm \infty} B(\xi, t)$. Note that $A_{ \pm}, B_{ \pm}$are positive and do not depend on $t$.

Lemma 4.1. Let $f_{1}$ be a forward shock wave solution of (1.1) with strength $\delta$, i.e. satisfying (1.6). Let $f_{2}$ be a corresponding diffusive wave solution of (2.19), (2.20), for initial data $f_{I}-f_{1}$ of magnitude $\delta$ i.e. satisfying (1.7). If $\delta$ is sufficiently small then

(i) There are positive constants $c, C$, and $\kappa$, independent of $x$, th and $\delta$, such that

$$
\begin{gathered}
c<A_{+}-\kappa \delta<A(\xi, t)<A_{-}+\kappa \delta<C, \\
c<B_{+}-\kappa \delta<B(\xi, t)<B_{-}+\kappa \delta<C, \quad s A_{\xi}<\kappa \delta .
\end{gathered}
$$

(ii) There is $\lambda>0$ such that $\sup _{\xi} D_{i}<0$ for $i=1,2,3$ in which

$$
\begin{gathered}
D_{1}=A(A-\lambda), \quad D_{2}=\lambda(\lambda-B), \\
D_{3}=M^{2}-4(A-\lambda)(\lambda-B) .
\end{gathered}
$$

(iii) Let $\lambda, \beta$ be constants. If $\beta$ is small enough then

$$
\begin{aligned}
&-\frac{\lambda}{2}\left(A^{-1}(M-s B)\right)_{\xi}-\left|\left(A^{-1}\right)_{t}\right|-\frac{\lambda}{2}\left[\left(A^{-1} B\right)_{t}+s\left(A^{-1}\right)_{\xi t}\right]-\beta\left|\left(A^{-1}\right)_{\xi}\right| \\
& \geqq\left\{\begin{array}{lll}
-\kappa \delta e^{-\gamma t} & \text { for } & \xi>0 \\
-\kappa\left|\lambda_{1 x}\right| & \text { for } & \xi<0
\end{array}\right.
\end{aligned}
$$

for some constant $\kappa$.

Proof. This lemma was proved in [6] for the case of a shock, i.e. $f_{2} \equiv 0$, with $\kappa=0$ in (i), (iii). In that case $\partial_{t} \equiv 0$. Since $\left|f_{2}\right|<c \delta$, the inequalities (4.9), (ii) are only slightly perturbed. Since $\left|f_{2 \xi}\right|+\left|f_{2_{t}}\right| \leqq c \delta e^{-\gamma t}$ for $\xi>0$ and $\left|f_{2 \xi}\right|+\left|f_{2 t}\right| \leqq c\left|\lambda_{1 x}\right|$ for $\xi<0$ the inequality (4.10) is derived.

The second lemma describes bounds on $H, K$, and $\Gamma$.

Lemma 4.2. Let $H=H_{1}+H_{2}+H_{3}, K, \Gamma$ be defined by (3.12)-(3.14), (3.19), (3.10) Denote

$$
F=\left(|\phi|+\left|\phi_{\xi}\right|+\left|\phi_{\xi \xi}\right|+|\psi|+\left|\psi_{\xi}\right|+\left|\psi_{\xi \xi}\right|+\left|\psi_{t}\right|+\left|\psi_{t \xi}\right|\right) \text {. }
$$

Then

$$
\begin{gathered}
\int_{0}^{\infty} \int\left(|H|+\left|H_{\xi}\right|\right) F d \xi d t \leqq c \delta N_{1}(t), \\
\int_{0}^{\infty} \int\left(|K|+\left|K_{\xi}\right|\right) F d \xi d t \leqq c \delta N_{1}(t) N_{2}(t), \\
\iint\left(|\Gamma|+\left|\Gamma_{\xi}\right|\right) F d \xi d t \leqq c N_{1}(t) N_{2}(t)^{2} .
\end{gathered}
$$


Proof of Lemma 4.2. First derive pointwise bounds on $H_{i}, K$, and $\Gamma$. For $H_{1}$ use (3.15), (2.32-2.35), (2.38) to obtain

$$
\begin{aligned}
\left|H_{1}\right|+\left|H_{1 \xi}\right| & \leqq c\left(\left|e_{1}\right|+\left|e_{2}\right|+\left|u_{2 x x}\right|+\left|u_{2 x t}\right|+\left|u_{2 x}\right|^{2}+\left|u_{2 x} u_{2 t}\right|\right) \\
& \leqq c \delta(1+t)^{-3 / 2}\left(|y|^{3}+1\right) e^{-y^{2}}
\end{aligned}
$$

in which $y=(x-\lambda t) / 2 \sqrt{\alpha t}$. In estimating $H_{2}$, use (3.13) and the fact that $f_{1}-f_{1}^{-\infty}$ and $f_{2}$ have nearly disjoint supports as seen from (2.8) and (2.31), so that

$$
\begin{aligned}
\left|H_{2}\right|+\left|H_{2 \xi}\right| & \leqq c\left|f_{2}\right|\left|f_{1}-f_{1}^{-\infty}\right| \leqq c \delta^{2}(t+1)^{-1 / 2} e^{-y^{2}}(\tanh \kappa(x-s t)+1) \\
& \leqq c \delta^{2} e^{-c(|x|+t)} .
\end{aligned}
$$

For $H_{3}$ use (3.14), (2.44), (2.45) to estimate

$$
\begin{gathered}
\left|H_{3}\right|+\left|H_{3 \xi}\right| \leqq\left|f_{3}\right|^{2}+\left|f_{3 x}\right|+\left|f_{3 t}\right| \\
\leqq c \delta\left\{\left[1+t+t y^{2}\right]^{-3 / 2}+\left[1+t+t^{1 / 2}|y|\right]^{-2}\right. \\
\left.\quad+|t+1|^{-3 / 2}\left(\left|f_{1 x}\right|+\left|f_{2 x}\right|\right)\right\} .
\end{gathered}
$$

Estimate $K$ using (3.19), (2.44), (2.45) to obtain

$$
|K|+\left|K_{\xi}\right|<\left(\left|f_{3}\right|+\left|f_{3 x}\right|\right)\left(\left|\phi_{x}\right|+\left|\psi_{x}\right|+\left|\psi_{t}\right|+\left|\phi_{x x}\right|+\left|\psi_{x x}\right|+\left|\psi_{x t}\right|\right)
$$

Finally estimate $\Gamma=\Gamma\left(\phi_{\xi}, \psi_{\xi},-\psi_{t}+s \psi_{\xi}\right)$ from its definition (3.10) as

$$
|\Gamma|+\left|\Gamma_{\xi}\right|<\left(\left|\phi_{\xi}\right|+\left|\psi_{\xi}\right|+\left|\psi_{t}\right|\right)\left(\left|\phi_{\xi}\right|+\left|\phi_{\xi \xi}\right|+\left|\psi_{\xi}\right|+\left|\psi_{\xi \xi}\right|+\left|\psi_{t}\right|+\left|\psi_{t \xi}\right|\right) .
$$

It follows that

$$
\begin{aligned}
& \int_{0}^{t} \int\left(|H|+\left|H_{\xi}\right|\right) F d \xi d \tau \leqq\left(\sup _{0 \leqq \tau \leqq t}\left(\int F^{2} d \xi\right)^{1 / 2}\right) \int_{0}^{\infty}\left(\int H^{2}+H_{\xi}^{2} d \xi\right)^{1 / 2} d \tau \leqq c \delta N_{1}(t) \\
& \int_{0}^{t} \int\left(|K|+\left|K_{\xi}\right|\right) F d \xi d \tau \\
& \leqq \int_{0}^{t} \int F\left(\left|f_{3}\right|+\left|f_{3 \xi}\right|\right)\left(\left|\phi_{\xi}\right|+\left|\phi_{\xi \xi}\right|+\left|\psi_{\xi}\right|+\left|\psi_{\xi \xi}\right|+\left|\psi_{t}\right|+\left|\psi_{t \xi}\right|\right) d \xi d \tau \\
& \leqq \int_{0}^{t} \sup _{\xi}\left(\left|f_{3}\right|+\left|f_{3 \xi}\right|\right)\left\|\phi_{\xi}, \psi_{\xi}, \psi_{t}\right\|_{1}\left\|\phi, \psi, \psi_{t}, \phi_{\xi}, \psi_{\xi}\right\|_{1} d \tau \\
& \leqq\left(\int_{0}^{t} \sup _{\xi}\left(\left|f_{3}\right|+\left|f_{3 \xi}\right|\right)^{2} d \tau\right)^{1 / 2} N_{1}(t)\left(\sup _{0 \leqq \tau \leqq t}\left\|\phi, \psi, \psi_{t}, \phi_{\xi}, \psi_{\xi}\right\|_{1}\right) \\
& \leqq c \delta N_{1}(t) N_{2}(t) \\
& \int_{0}^{t} \int\left(|\Gamma|+\left|\Gamma_{\xi}\right|\right) F d \xi d \tau \leqq \int_{0}^{t} \int\left(|\phi|+\left|\phi_{\xi}\right|+|\psi|+\left|\psi_{\xi}\right|+\left|\psi_{t}\right|\right) \\
& \times\left(\left|\phi_{\xi}\right|+\left|\phi_{\xi \xi}\right|+\left|\psi_{\xi}\right|+\left|\psi_{\xi \xi}\right|+\left|\psi_{t}\right|+\left|\psi_{t \xi}\right|\right)^{2} d \xi d \tau \\
& \leqq \sup _{\xi .0 \leqq \tau \leqq t}\left(|\phi|+\left|\phi_{\xi}\right|+|\psi|+\left|\psi_{\xi}\right|+\left|\psi_{t}\right|\right) N_{2}(t)^{2} \leqq c N_{1}(t) N_{2}(t)^{2} \text {, }
\end{aligned}
$$

which concludes the proof of the lemma. 
Now we proceed with the energy estimates, following the analysis of Kawashima and Matsumura [6]. There are four such estimates presented in Lemma 4.3 ; they are analogous to Lemmas 3.5-3.7 of [6].

Lemma 4.3 (Preliminary energy estimates). Let $\phi, \psi$ solve (3.17), (3.18) with $\delta$ sufficiently small. Then for some constant $c$

i) $\|\phi(t)\|^{2}+\|\psi(t)\|_{1}^{2}+\left\|\psi_{t}(t)\right\|^{2}+\int_{0}^{t}\left\|\psi_{\xi}, \psi_{t}\right\|^{2} d \tau$

$$
\begin{aligned}
\leqq & c\left(\|\phi(0)\|^{2}+\|\psi(0)\|_{1}^{2}+\left\|\psi_{t}(0)\right\|^{2}+\iint_{\Omega_{+}(t)}\left|\lambda_{1 x}\right| \psi^{2} d x d \tau\right. \\
& \left.+\delta N_{1}(t)^{2}+\delta N_{1}(t)+\delta N_{1}(t) N_{2}(t)+N_{1}(t) N_{2}(t)^{2}\right),
\end{aligned}
$$

ii) $\left\|\phi_{\xi}(t)\right\|^{2}+\int_{0}^{t}\left\|\phi_{\xi}\right\|^{2} d \tau-c\left(\left\|\psi_{\xi}, \psi_{t}(t)\right\|^{2}+\int_{0}^{t}\left\|\psi_{\xi}, \psi_{t}\right\|^{2} d \tau\right)$

$$
\leqq c\left(\left\|\phi_{\xi}(0)\right\|^{2}+\left\|\psi_{t}(0)\right\|^{2}+\delta N_{1}(t)+\delta N_{1}(t) N_{2}(t)+N_{1}(t) N_{2}(t)^{2}\right),
$$

iii) $\left\|\phi_{\xi}(t)\right\|^{2}+\left\|\psi_{\xi}(t)\right\|_{1}^{2}+\left\|\psi_{t \xi}(t)\right\|^{2}+\int_{0}^{t}\left\|\psi_{t \xi}, \psi_{\xi \xi}\right\|^{2} d \tau-c \int_{0}^{t}\left\|\phi_{\xi}, \psi_{\xi}, \psi_{t}\right\|^{2} d \tau$

$$
\begin{aligned}
\leqq & c\left(\left\|\phi_{\xi}(0)\right\|^{2}+\left\|\psi_{\xi}(0)\right\|_{1}^{2}+\left\|\psi_{t \xi}(0)\right\|^{2}\right. \\
& \left.+\delta N_{1}(t)+\delta N_{1}(t) N_{2}(t)+N_{1}(t) N_{2}(t)^{2}\right),
\end{aligned}
$$

iv) $\left\|\phi_{\xi \xi}(t)\right\|^{2}+\int_{0}^{t}\left\|\phi_{\xi \xi}\right\|^{2} d \tau-c\left(\left\|\psi_{\xi \xi}, \psi_{t \xi}(t)\right\|^{2}+\int_{0}^{t}\left(\left\|\phi_{\xi}\right\|^{2}+\left\|\psi_{\xi}, \psi_{t}\right\|_{1}^{2}\right) d \tau\right)$

$$
\leqq c\left(\left\|\phi_{\xi \xi}(0)\right\|^{2}+\left\|\psi_{t \xi}(0)\right\|^{2}+\delta N_{1}(t)+\delta N_{1}(t) N_{2}(t)+N_{1}(t) N_{2}(t)^{2}\right) .
$$

Note that $\psi_{t}(0)=-z_{*}(0)$. A suitable linear combination of the four estimates (4.23)-(4.26) results in the main estimate of this section.

Lemma 4.4 (Principal energy estimate). Under the assumptions of Lemma 4.3,

$$
\begin{aligned}
N_{1}(t)\left(N_{1}(t)-c \delta-c \delta N_{2}(t)-N_{2}(t)^{2}\right)+N_{2}(t)^{2} \leqq & c N_{1}(0)^{2} \\
& +\int_{0}^{t} \int_{\Omega+(t)}\left|\lambda_{1 x}\right| \psi^{2} d x d \tau .
\end{aligned}
$$

The norms $N_{1}, N_{2}$ are defined in (4.5), (4.6). The proof of Lemma 4.4 is immediate. Bounding the integral on the right of (4.27) is the object of Sect. 5.

Proof of Lemma 4.3.

(i) Proof of (4.23). Following [6] define

$$
\begin{aligned}
& \mathrm{LHS}_{1} \equiv-\psi_{\xi} L_{1}+A^{-1}\left(\psi_{t}-s \psi_{\xi}\right) L_{2}=-A^{-1}\left(\psi_{t}-s \psi_{\xi}\right) \Gamma_{1}, \\
& \mathrm{LHS}_{2} \equiv \phi L_{1}+A^{-1} \psi L_{2}=-A^{-1} \psi \Gamma_{1} .
\end{aligned}
$$

Integrate over $-\infty<\xi<\infty$, dropping some terms through integration by parts. After some rearrangements, one finds that

$$
\begin{aligned}
\mathrm{LHS}_{1}= & {\left[A^{-1}\left\{\frac{1}{2}\left(\psi_{t}-s \psi_{\xi}\right)^{2}+\frac{1}{2} \psi_{\xi}^{2}\right\}-\phi \psi_{\xi}\right]_{t} } \\
& +A^{-1}\left[B\left(\psi_{t}-s \psi_{\xi}\right)^{2}+M\left(\psi_{t}-s \psi_{\xi}\right) \psi_{\xi}-A \psi_{\xi}^{2}\right] \\
& +\frac{1}{2}\left(A^{-1}\right)_{\xi}\left[s\left(\psi_{t}-s \psi_{\xi}\right)^{2}+2 \psi_{t} \psi_{\xi}-s \psi_{\xi}^{2}\right]-\left(A^{-1}\right)_{t}\left[\frac{1}{2}\left(\psi_{t}-s \psi_{\xi}\right)^{2}+\frac{1}{2} \psi_{\xi}^{2}\right]+[]_{\xi},
\end{aligned}
$$




$$
\begin{aligned}
\operatorname{LHS}_{2}= & {\left[\frac{1}{2} \phi^{2}+\frac{1}{2} A^{-1} B \psi^{2}+A^{-1} \psi\left(\psi_{t}-s \psi_{\xi}\right)+\frac{1}{2} s\left(A^{-1}\right)_{\xi} \psi^{2}\right]_{t} } \\
& +A^{-1}\left[-\left(\psi_{t}-s \psi_{\xi}\right)^{2}+\psi_{\xi}^{2}\right]+\left(1-s^{2}\right)\left(A^{-1}\right)_{\xi} \psi \psi_{\xi}-\frac{1}{2}\left(A^{-1}(M-s B)\right)_{\xi} \psi^{2} \\
& -\left[\frac{1}{2}\left(A^{-1} B\right)_{t} \psi^{2}+\left(A^{-1}\right)_{t} \psi\left(\psi_{t}-s \psi_{\xi}\right)+\frac{1}{2} s\left(A^{-1}\right)_{\xi t} \psi^{2}\right]+[]_{\xi} .
\end{aligned}
$$

Add $\mathrm{LHS}_{1}+\lambda \mathrm{LHS}_{2}$ (here $\lambda$ is unrelated to the eigenvalues $\lambda_{i}$ ) to obtain

$$
\begin{aligned}
& \left(E_{1}+E_{2}+\tilde{E}_{2}\right)_{t}+E_{3}+E_{4}+E_{5}+E_{6}+G+[]_{\xi} \\
& \quad=-A^{-1}\left(\lambda \psi+\left(\psi_{t}-s \psi_{\xi}\right)\right) \Gamma_{1}
\end{aligned}
$$

in which

$$
\begin{aligned}
E_{1} & =A^{-1}\left(\frac{\lambda}{2} A \phi^{2}-A \phi \psi_{\xi}+\frac{1}{2} \psi_{\xi}^{2}\right), \\
E_{2} & =A^{-1}\left(\frac{\lambda}{2} B \psi^{2}+\lambda \psi\left(\psi_{t}-s \psi_{\xi}\right)+\frac{1}{2}\left(\psi_{t}-s \psi_{\xi}\right)^{2}\right), \\
\tilde{E}_{2} & =\frac{\lambda s}{2}\left(A^{-1}\right)_{\xi} \psi^{2} \\
E_{3} & =A^{-1}\left((B-\lambda)\left(\psi_{t}-s \psi_{\xi}\right)^{2}+M\left(\psi_{t}-s \psi_{\xi}\right) \psi_{\xi}+(\lambda-A) \psi_{\xi}^{2}\right), \\
E_{4} & =-\frac{\lambda}{2}\left(A^{-1}(M-s B)\right)_{\xi} \psi^{2}, \\
E_{5} & =-\left(A^{-1}\right)_{t}\left[\frac{1}{2}\left(\psi_{t}-s \psi_{\xi}\right)^{2}+\frac{1}{2} \psi_{\xi}^{2}-\lambda \psi\left(\psi_{t}-s \psi_{\xi}\right)\right], \\
E_{6} & =-\frac{\lambda}{2}\left[\left(A^{-1} B\right)_{t}+s\left(A^{-1}\right)_{\xi t}\right] \psi^{2}, \\
G & =\lambda\left(1-s^{2}\right)\left(A^{-1}\right)_{\xi} \psi \psi_{\xi}+\frac{1}{2}\left(A^{-1}\right)_{\xi}\left\{s\left(\psi_{t}-s \psi_{\xi}\right)^{2}+2\left(\psi_{t}-s \psi_{\xi}\right) \psi_{\xi}+s \psi_{\xi}^{2}\right\} .
\end{aligned}
$$

The quantities $E_{1}, E_{2}, \widetilde{E}_{2}, E_{3}, E_{4}, G$ are the same as in [6] except that $A, B, M$ depend on $f_{2}$ as well as $f_{1}$, and hence on $t$ as well as $\xi$.

It follows as in [6] from Lemma 4.1 that

$$
\begin{gathered}
E_{1}+E_{2}+\widetilde{E}_{2} \leqq C\left(\phi^{2}+\psi^{2}+\psi_{\xi}^{2}+\left(\psi_{t}-s \psi_{\xi}^{2}\right)\right), \\
E_{1}+E_{2}+\widetilde{E}_{2} \geqq c\left(\phi^{2}+\psi^{2}+\psi_{\xi}^{2}+\left(\psi_{t}-s \psi_{\xi}\right)^{2}\right), \\
E_{3} \geqq c\left(\psi_{\xi}^{2}+\left(\psi_{t}-s \psi_{\xi}\right)^{2}\right) .
\end{gathered}
$$

Since $\left|\left(A^{-1}\right)_{\xi}\right|+\left|\left(A^{-1}\right)_{t}\right|<\left(\left|\lambda_{1 x}\right|+\left|\lambda_{2 x}\right|\right)<\delta$, then

$$
\begin{aligned}
|G| & \leqq \beta\left|\left(A^{-1}\right)_{\xi}\right| \psi^{2}+c \beta^{-1} \delta\left(\psi_{\xi}^{2}+\left(\psi_{t}-s \psi_{\xi}\right)^{2}\right), \\
\left|E_{5}\right| & \leqq c \delta\left(\left(\psi_{t}-s \psi_{\xi}\right)^{2}+\psi_{\xi}^{2}\right)+\left|\left(A^{-1}\right)_{t}\right| \psi^{2},
\end{aligned}
$$

for any small $\beta>0$. By choosing $\delta$ and $\beta$ small enough, it follows using (iii) in Lemma 4.1 that

$$
E_{3}+E_{4}+E_{5}+E_{6}+G \geqq c\left(\left(\psi_{t}-s \psi_{\xi}\right)^{2}+\psi_{\xi}^{2}\right)-c\left(\chi_{+}\left|\lambda_{1 x}\right|+\delta e^{-\gamma t}\right) \psi^{2},
$$

in which $\chi_{+}=0$ for $x \in \Omega_{-} \cup \Omega_{2}, \chi_{+}=1$ for $x \in \Omega_{+}$. 
Now integrate the various terms in (4.31) over $-\infty<\xi<\infty$ and $0<\tau<t$ to obtain

$$
\begin{gathered}
\int_{0}^{t} \int\left(E_{1}+E_{2}+\widetilde{E}_{2}\right)_{t} d \xi d t \geqq c\left[\int \phi^{2}+\psi^{2}+\psi_{\xi}^{2}+\left(\psi_{t}-s \psi_{\xi}\right)^{2} \int\right]_{0}^{t}, \\
\int_{0}^{t} \int\left(E_{3}+E_{4}+E_{5}+E_{6}+G\right) d \xi d t \geqq c \int_{0}^{t} \int\left(\left(\psi_{t}-s \psi_{\xi}\right)^{2}+\psi_{\xi}^{2}\right) d \xi d t \\
-c \delta N_{1}(t)^{2}-c \int_{0}^{t} \int_{\Omega+(t)}\left|\lambda_{1 x}\right| \psi^{2} d x d t, \\
\int_{0}^{t} \int\left|A^{-1}\left(\lambda \psi+\left(\psi_{t}-s \psi_{\xi}\right)\right) \Gamma_{1}\right| d \xi d t \leqq c \int_{0}^{t} \int(|\Gamma|+|K|+|H|) F d \xi d t \\
\leqq c\left(\delta N_{1}(t)+\delta N_{1}(t) N_{2}(t)+N_{1}(t) N_{2}(t)^{2}\right) .
\end{gathered}
$$

Combine these together to obtain (4.23).

(ii) Proof of (4.24)-(4.26). These estimates are proved as in [6] and (i) with very little change.

\section{Characteristic Energy Method}

The object here is to estimate $N_{3}(t)^{2}=\int_{0}^{t} \int\left(\left|\lambda_{1 x}\right|+\left|\lambda_{2 x}\right|\right)\left(\phi^{2}+\psi^{2}\right) d x d t$ on the righthand side of the energy estimate (4.27) in Lemma 4.4. Rewrite the system (3.17), (3.18) for $\phi, \psi$ by diagonalizing the left-hand side. As in (2.25), (2.26) the convection matrix

$$
\left[\begin{array}{cc}
0 & 1 \\
A / B & M / B
\end{array}\right]
$$

has right eigenvectors $r_{1}, r_{2}$, left eigenvectors $l_{1}, l_{2}$ and eigenvalues $\lambda_{1}, \lambda_{2}$ given by

$$
\begin{array}{cc}
r_{1}=(-B / D,-(M-D) / 2 D)^{\dagger}, & r_{2}=(B / D,(M+D) / 2 D)^{\dagger}, \\
l_{1}=(-(M+D) / 2 B, 1), & l_{2}=((-M+D) / 2 B, 1), \\
\lambda_{1}=(M-D) / 2 B, & \lambda_{2}=(M+D) / 2 B,
\end{array}
$$

in which $D=\left(M^{2}+4 A B\right)^{1 / 2}$ and $M, A, B$ are defined in (2.41) and depend only on $f_{1}$ and $f_{2}$. Define characteristic variables $\theta_{1}, \theta_{2}$ by

which satisfy

$$
\left(\begin{array}{l}
\phi \\
\psi
\end{array}\right)=\theta_{1} r_{1}+\theta_{2} r_{2},
$$

$$
\begin{aligned}
c\left(\phi^{2}+\psi^{2}\right) & <\theta_{1}^{2}+\theta_{2}^{2}<C\left(\phi^{2}+\psi^{2}\right), \\
c\left(\phi_{x}^{2}+\psi_{x}^{2}\right) & <\theta_{1 x}^{2}+\theta_{2 x}^{2}+\left(\left|\lambda_{1 x}\right|+\left|\lambda_{2 x}\right|\right)\left(\theta_{1}^{2}+\theta_{2}^{2}\right) \\
& <C\left(\phi_{x}^{2}+\psi_{x}^{2}\right)+C\left(\left|\lambda_{1 x}\right|+\left|\lambda_{2 x}\right|\right)\left(\phi^{2}+\psi^{2}\right),
\end{aligned}
$$

etc. for some constants $c, C$ independent of $x, t, \delta$. The characteristic form for (3.17), (3.18) is

$$
\theta_{i t}+\lambda_{i} \theta_{i x}=\sum_{k=1}^{2} \theta_{k}\left(l_{l t}+\lambda_{i} l_{l x}\right) \cdot r_{k}+B^{-1}\left(\psi_{x x}-\psi_{t t}-\Gamma-K-H\right) .
$$


We first obtain an energy estimate from the characteristic form:

Lemma 5.1. For $\delta$ small enough,

$$
\begin{aligned}
& \int|\theta(x, t)|^{2} d x+\frac{1}{2} \iint_{\Omega_{2}(t)}\left|\lambda_{2 x}\right| \theta_{2}^{2} d x d \tau+\frac{1}{2} \iint_{\Omega_{-}(t)}\left|\lambda_{1 x}\right| \theta_{1}^{2} d x d \tau \\
& \leqq C\left\{\sum_{i \neq j} \iint_{\Omega_{j}(t)}\left|\lambda_{j x}\right| \theta_{i}^{2} d x d \tau+\iint_{\Omega_{+}(t)}\left|\lambda_{1 x}\right| \theta_{1}^{2} d x d \tau\right. \\
&\left.+N_{1}(t)\left\{\delta+N_{1}(t)+\delta N_{2}(t)+N_{2}(t)^{2}\right\}+N_{2}(t)^{2}\right\} .
\end{aligned}
$$

Proof of Lemma 5.1. Multiply the characteristic form equation (5.5) by $\theta_{i}$ and integrate over $-\infty<x<\infty, 0<\tau<t$,

$$
\begin{gathered}
\int \theta_{i}^{2}(x, t) d x-\int_{0}^{t} \int \lambda_{i x} \theta_{i}^{2} d x d \tau=\int \theta_{i}^{2}(x, 0) d x \\
+2 \int_{0}^{t} \int\left[\theta_{i} \sum_{k=1}^{2} \theta_{k}\left(l_{i t}+\lambda_{i} l_{i x}\right) \cdot r_{k}+\theta_{i} B^{-1}\left(\psi_{x x}-\psi_{t t}-\Gamma-K-H\right)\right] d x d \tau .
\end{gathered}
$$

Throughout this analysis we use the fact that $\lambda_{i}$ depends primarily on $f_{1}$ in $\Omega_{2}$ (i.e. $x>0$ ) and on $f_{2}$ in $\Omega_{1}$ (i.e. $x<0$ ).

First we partly handle the second term on the left by noting that, from (2.17) for $\Omega_{2}$ and (2.29) for $\Omega_{-}$and for some $\gamma$,

$$
\begin{array}{ll}
-\lambda_{1 x}=-\lambda_{1 x}\left(f_{2}\right)+O\left(f_{1 x}\right)=\left|\lambda_{1 x}\right|+O\left(\delta e^{-\gamma(|x|+t)}\right) & \text { for } \quad(x, t) \in \Omega_{-}, \\
-\lambda_{2 x}=-\lambda_{2 x}\left(f_{1}\right)+O\left(f_{2 x}\right)=\left|\lambda_{2 x}\right|+O\left(\delta e^{-\gamma(|x|+t)}\right) & \text { for } \quad(x, t) \in \Omega_{2} .
\end{array}
$$

Thus

$$
\begin{aligned}
\int_{0}^{t} \int \lambda_{1 x} \theta_{1}^{2} & +\lambda_{2 x} \theta_{2}^{2} d x d \tau \leqq-\iint_{\Omega_{-}(t)}\left|\lambda_{1 x}\right| \theta_{1}^{2} d x d \tau-\iint_{\Omega_{2}}\left|\lambda_{2 x}\right| \theta_{2}^{2} d x d \tau \\
& +\iint_{\Omega_{+}(t)}\left|\lambda_{1 x}\right| \theta_{1}^{2} d x d \tau+c \delta N_{1}(t)^{2} .
\end{aligned}
$$

Next use the bounds

$$
\begin{array}{ll}
l_{i t}+\lambda_{i} l_{i x}=O(1) \lambda_{i x x}+O\left(\delta e^{-\gamma(|x|+t)}\right) & \text { on } \quad \Omega_{i}, \\
\left|l_{i t}+\lambda_{i} l_{i x}\right| \leqq c\left|\lambda_{j x}\right|+O\left(\delta e^{-\gamma(|x|+t)}\right) & \text { on } \quad \Omega_{j}(i \neq j),
\end{array}
$$

which follow from (2.18), (2.36), (2.37), to estimate (integrating by parts)

$$
\begin{gathered}
\left|\int_{\Omega_{2}(t)} \theta_{i} \sum_{k=1}^{2} \theta_{k}\left(l_{i t}+\lambda_{i} l_{i x}\right) \cdot r_{k} d x d t\right| \leqq c \delta\left(\iint_{\Omega_{i}(t)} \theta^{2}\left|\lambda_{i x}\right| d x d t+N_{1}(t)^{2}\right), \\
\left|\int_{\Omega_{j}(t)} \theta_{i} \sum_{k=1}^{2} \theta_{k}\left(l_{i t}+\lambda_{i} l_{i x}\right) \cdot r_{k} d x d t\right| \\
\leqq \frac{1}{4} \iint_{\Omega_{j}(t)} \theta_{j}^{2}\left|\lambda_{j x}\right| d x d t+c \iint_{\Omega_{j}(t)} \theta_{i}^{2}\left|\lambda_{j x}\right| d x d t+c \delta N_{1}(t)^{2},
\end{gathered}
$$

for some constant $c$.

Next since $B^{-1}$ is bounded,

$$
\int_{0}^{t} \int \theta_{i} B^{-1}(\Gamma+K+H) d x d t \leqq c\left(\delta+\delta N_{2}(t)+N_{2}(t)^{2}\right) N_{1}(l)
$$


from Lemma 4.2. Finally use $\left|B_{x}\right|+\left|B_{t}\right|<c\left(\left|\lambda_{1 x}\right|+\left|\lambda_{2 x}\right|\right)<c \delta$ and $\phi_{x}=-\psi_{t}$ to estimate

$$
\begin{aligned}
\left|\int_{0}^{t} \int \theta_{i} B^{-1}\left(\psi_{x x}-\psi_{t t}\right) d x d t\right| \leqq & c\left(\int_{0}^{t} \int \theta_{x}^{2}+\theta_{t}^{2}+\left(\left|\lambda_{1 x}\right|+\left|\lambda_{2 x}\right|\right)\left(\left|\theta \theta_{x}\right|+\left|\theta \theta_{t}\right| d x d t\right.\right. \\
& \left.+\int\left|\theta \theta_{t}(t)\right|+\left|\theta \theta_{t}\right|(0) d x\right) \\
\leqq & c\left(N_{2}(t)^{2}+\delta \iint\left(\left|\lambda_{1 x}\right|+\left|\lambda_{2 x}\right|\right) \theta^{2} d x d t+N_{1}(t)^{2}\right)(5.1
\end{aligned}
$$

Combine (5.7), (5.10), (5.12)-(5.15) to obtain the desired estimate (5.6) and complete the proof of Lemma 5.1.

Since $N_{1}$ and $N_{2}$ can be bounded through the energy estimate (4.27), we now only need to find bounds on the three integrals on the right of (5.6):

$$
\begin{gathered}
I_{1}=\iint_{\Omega_{1}(t)}\left|\lambda_{1 x}\right| \theta_{2}^{2} d x d \tau \\
I_{2}=\iint_{\Omega_{2}(t)}\left|\lambda_{2 x}\right| \theta_{1}^{2} d x d \tau \text { and } I_{3}=\iint_{\Omega_{+}(t)}\left|\lambda_{1 x}\right| \theta_{1}^{2} d x d \tau .
\end{gathered}
$$

The estimates are derived using the characteristic energy method developed by Liu [7]. Actually we use a somewhat simplified version in which integration is performed along piecewise linear approximations of the characteristics. Although simpler, this version is less robust since its validity depends in an additional way on the shock being weak.

Write the eigenvalue $\lambda_{i}$ as piecewise constant part $\bar{\lambda}_{i}$ plus an error $\tilde{\lambda}_{i}$, i.e.

$$
\lambda_{i}=\bar{\lambda}_{i}+\tilde{\lambda}_{i}
$$

in which

$$
\bar{\lambda}_{i}(x, t)= \begin{cases}\lambda_{i}\left(f_{1}^{\infty}\right)=\lambda_{i}^{+}, & x>s t \\ \lambda_{i}\left(f_{1}^{-\infty}\right)=\lambda_{i}^{-}, & x<s t .\end{cases}
$$

Define approximate, piecewise linear characteristics $X_{1}(\tau, t, x), X_{2}(\tau, t, x)$ (cf. Fig. 2) satisfying

$$
\frac{\partial}{\partial \tau} X_{i}(\tau, t, x)=\bar{\lambda}_{i}\left(X_{i}, \tau\right), \quad X_{i}(t, t, x)=x
$$

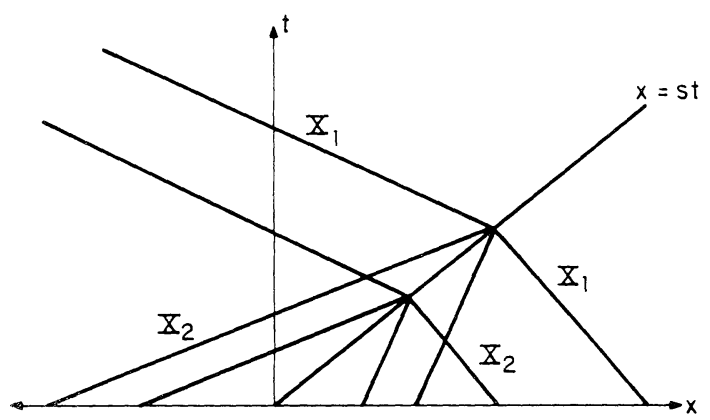

Fig. 2. Piecewise linear characteristics $X_{1}$ and $X_{2}$ 
i.e.

$$
\begin{gathered}
X_{1}(\tau, t, x)= \begin{cases}x+\lambda_{1}^{+}(\tau-t) & \text { if } x>s t, X_{1}>s \tau \\
x+\lambda_{1}^{-}(\tau-t) & \text { if } x<s t, X_{1}<s \tau \\
\frac{\left(s-\lambda_{1}^{-}\right)}{\left(s-\lambda_{1}^{+}\right)}\left(x-\lambda_{1}^{+} t\right)+\lambda_{1}^{-} \tau & \text { if } x>s t, X_{1}<s \tau\end{cases} \\
X_{2}(\tau, t, x)= \begin{cases}x+\lambda_{2}^{+}(\tau-t) & \text { if } x>s t \\
x+\lambda_{2}^{-}(\tau-t) & \text { if } x<s t .\end{cases}
\end{gathered}
$$

Note that $X_{2}$ is not defined past the time that it intersects $X_{2}=s \tau$. Equation (5.5) can be rewritten as

$$
\theta_{i t}+\bar{\lambda}_{i} \theta_{i x}=\sum_{k=1}^{2} \theta_{k}\left(l_{i t}+\lambda_{i} l_{i x}\right) \cdot r_{k}+B^{-1}\left(\psi_{x x}-\psi_{t t}-\Gamma-K-H\right)-\tilde{\lambda}_{i} \theta_{i x} .
$$

Multiply by $\theta_{i}$ and integrate along $X_{i}$ to obtain

$$
\begin{aligned}
\theta_{i}(x, t)^{2}= & \frac{1}{2} \int_{0}^{t} \theta_{i}\left\{\sum_{k=1}^{2} \theta_{k}\left(l_{i t}+\lambda_{i} l_{i x}\right) \cdot r_{k}\right. \\
& \left.+B^{-1}\left(\psi_{x x}-\psi_{t t}-\Gamma-K-H\right)-\tilde{\lambda}_{i} \theta_{i x}\right\}\left(X_{i}(\tau, t, x), \tau\right) d \tau+\theta_{i}\left(X_{i}(0, t, x), 0\right)^{2} .
\end{aligned}
$$

For any non-negative function $g(x, t)$ we integrate to get

$$
\begin{aligned}
& \int_{0}^{t} \int g \theta_{i}^{2} d x d \tau=\frac{1}{2} \int_{0}^{t} \int \tilde{G} \theta_{i}\left\{\sum_{k=1}^{2} \theta_{k}\left(l_{i t}+\lambda_{i} l_{i x}\right) \cdot r_{k}\right. \\
& \left.\quad+B^{-1}\left(\psi_{x x}-\psi_{t t}-\Gamma-K-H\right)-\tilde{\lambda}_{i} \theta_{i x}\right\}(x, \tau) d x d \tau+\int_{-\infty}^{\infty}\left(\widetilde{G} \theta_{i}^{2}\right)(x, 0) d x \\
& \leqq \\
& \frac{1}{2} \int_{0}^{t} \int G \theta_{i}\left\{\sum_{k=1}^{2} \theta_{k}\left(l_{i t}+\lambda_{i} l_{i x}\right) \cdot r_{k}+B^{-1}\left(\psi_{x x}-\psi_{t t}-\Gamma-K-H\right)-\tilde{\lambda}_{i} \theta_{i x}\right\} \\
& \quad+\int_{-\infty}^{\infty}\left(G \theta_{i}^{2}\right)(x, 0) d x
\end{aligned}
$$

in which

$$
\begin{aligned}
\widetilde{G}(x, \tau) & =\int_{\tau}^{t} g\left(X_{i}(\sigma, \tau, x), \sigma\right) \frac{\partial X_{i}}{\partial x}(\sigma, \tau, x) d \sigma, \\
G(x, \tau) & =\int_{\tau}^{\infty} g\left(X_{i}(\sigma, \tau, x), \sigma\right) \frac{\partial X_{i}}{\partial x}(\sigma, \tau, x) d \sigma .
\end{aligned}
$$

The inequality in (5.20) follows from the non-negativity of $g$ and $\left(\partial X_{i} / \partial x\right)$. 
Since $X_{2}$ can only be extended forward for a finite time, until it hits the line $x^{\prime}=s t^{\prime}$, define $\partial X_{2} / \partial x=0$ for $\sigma$ past that time. Note that $\left(\frac{\partial X_{i}}{\partial x}\right)^{-1}$ is piecewise
constant and bounded, i.e. for $\sigma>\tau, X_{i}(\sigma, \tau, x)$ satisfies

$$
\begin{aligned}
& \frac{\partial X_{1}}{\partial x}=\left\{\begin{array}{lll}
1 & \text { for } \quad\left(x>s \tau, X_{1}>s \sigma\right) \\
\left(s-\lambda_{1}^{-}\right)\left(s-\lambda_{1}^{+}\right)^{-1} & \text { for } \quad x>s \tau, X_{1}<s \sigma, & \text { or } \quad\left(x<s \tau, X_{1}<s \sigma\right)
\end{array}\right. \\
& \frac{\partial X_{2}}{\partial x}(\sigma, \tau, x)=\left\{\begin{array}{rr}
1 & \text { for } \quad\left(x>s \tau, \sigma<\left(s-\lambda_{2}^{+}\right)^{-1}\left(x-\lambda_{2}^{+} \tau\right)\right) \\
& \left(x<s \tau, \sigma<\left(s-\lambda_{2}^{-}\right)^{-1}\left(x-\lambda_{2}^{-} \tau\right)\right)
\end{array}\right. \text { or }
\end{aligned}
$$

For three different choices of $g$ we shall utilize (5.20). For each choice the various terms on the right-hand side of (5.20) will be estimated as:

$$
\begin{aligned}
& \left|\int_{0}^{t} \int_{-\infty}^{\infty} \theta_{i} \theta_{k}\left(l_{i t}+\lambda_{i} l_{i x}\right) \cdot r_{k} G d x d \tau\right| \leqq(\sup |G|) \int_{0}^{t} \int_{-\infty}^{\infty}\left(\left|\lambda_{1 x}\right|+\left|\lambda_{2 x}\right|\right) \theta^{2} d x d \tau \\
& \leqq(\sup |G|) N_{3}(t)^{2} \\
& \left|\int_{0}^{t} \int_{-\infty}^{\infty} \tilde{\lambda}_{i} \theta_{i} \theta_{i x} G d x d \tau\right| \leqq\left(\int_{0}^{t} \int_{-\infty}^{\infty} \theta_{i x}^{2} d x d t\right)^{1 / 2}\left(\int_{0}^{t} \int_{-\infty}^{\infty}\left(\tilde{\lambda}_{i} G\right)^{2} \theta_{i}^{2} d x d \tau\right)^{1 / 2} \\
& \leqq c N_{2}(t)\left(\int_{0}^{t} \int_{-\infty}^{\infty}\left(\tilde{\lambda}_{i} G\right)^{2} \theta_{i}^{2} d x d \tau\right)^{1 / 2} \\
& \left|\int_{0}^{t} \int_{-\infty}^{\infty} G \theta_{i} B^{-1}(\Gamma+K+H) d x d \tau\right| \leqq c(\sup |G|) \int_{0}^{t} \int_{-\infty}^{\infty}\left|\theta_{i}\right|(|\Gamma|+|K|+|H|) d x d \tau \\
& \leqq c(\sup |G|) N_{1}(t)\left(\delta+\delta N_{2}(t)+N_{2}(t)^{2}\right) \text {, } \\
& \int_{-\infty}^{\infty}\left(G \theta_{i}^{2}\right)(x, 0) d x=(\sup |G|) N_{1}(0)^{2} \text {. }
\end{aligned}
$$

Since $G(x, t)$ has a jump discontinuity along the line $x=s t$, write

$$
\begin{gathered}
\frac{\partial}{\partial x} G=\bar{G}(x) \delta(x-s t)+G_{x}(x, t), \\
\frac{\partial}{\partial t} G=s^{-1} \bar{G}(x) \delta(x-s t)+G_{t}(x, t),
\end{gathered}
$$

in which $\bar{G}(x)$ is the size of the jump in $G$ at $\left(x, t=s^{-1} x\right)$ and $G_{x}, G_{t}$ are regular functions. We need a bound on integrals along the line $x=$ st. From Eq. (3.18), $\psi_{t t}^{2}<c\left(\phi_{x}^{2}+\psi_{x}^{2}+\psi_{t}^{2}+\psi_{x x}^{2}+H^{2}+\phi_{x}^{4}+\psi_{x}^{4}+\psi_{t}^{4}\right)$. Since $\int_{0}^{\infty} \int H^{2} d x d \tau<c \delta^{2} \quad$ and $\left|\phi_{x}\right|+\left|\psi_{x}\right|+\left|\psi_{t}\right|<N_{1}$, then the Sobolev inequality implies that

$$
\begin{aligned}
\int_{0}^{t}\left(\psi_{x}^{2}+\psi_{\tau}^{2}\right)(s \tau, \tau) d \tau^{1 / 2} & \leqq \int_{0}^{t} \int_{-\infty}^{\infty}\left(\psi_{x}^{2}+\psi_{t}^{2}+\psi_{x x}^{2}+\psi_{x t}^{2}+\psi_{t t}^{2}\right) d x d \tau^{1 / 2} \\
& \leqq c\left(\delta+N_{2}(t)+N_{1}(t) N_{2}(t)\right)
\end{aligned}
$$


Now we are ready [using (5.4)] to estimate the terms in (5.20) containing $\psi_{x x}, \psi_{t t}$ :

$$
\begin{aligned}
& \left|\int_{0}^{t} \int_{-\infty}^{\infty} G \theta_{i} B^{-1}\left(\psi_{x x}-\psi_{\tau \tau}\right) d x d \tau\right|=\mid-\int_{0}^{t} \int_{-\infty}^{\infty}\left[G B^{-1}\left(\theta_{i x} \psi_{x}-\theta_{i \tau} \psi_{\tau}\right)\right. \\
& \left.+G\left(\left(B^{-1}\right)_{x} \theta_{i} \psi_{x}-\left(B^{-1}\right)_{\tau} \theta_{i} \psi_{\tau}\right)+B^{-1}\left(G_{x} \theta_{i} \psi_{x}-G_{\tau} \theta_{i} \psi_{\tau}\right)\right] d x d \tau \\
& \left.-\int_{-\infty}^{\infty} G \theta_{i} B^{-1} \psi_{\tau} d x\right]_{0}^{t}-\int_{0}^{t} \bar{G} \theta_{i} B^{-1} \psi_{x}(s \tau, \tau) d \tau+\int_{0}^{s t} \bar{G} \theta_{i} B^{-1} \psi_{t}\left(x, s^{-1} x\right) d x \mid \\
& \leqq c(\sup |G|) \int_{0}^{t} \int_{-\infty}^{\infty} \psi_{x}^{2}+\psi_{\tau}^{2}+\phi_{x}^{2}+\phi_{\tau}^{2} d x d \tau \\
& +c(\sup |G|)\left(\int_{0}^{t} \int_{-\infty}^{\infty} \psi_{x}^{2}+\psi_{\tau}^{2} d x d \tau\right)^{1 / 2}\left(\int_{0}^{t} \int_{-\infty}^{\infty}\left(\left|\lambda_{1 x}\right|+\left|\lambda_{2 x}\right|\right) \theta^{2} d x d \tau\right)^{1 / 2} \\
& +c\left(\int_{0}^{t} \int_{-\infty}^{\infty} \psi_{x}^{2}+\psi_{\tau}^{2} d x d \tau\right)^{1 / 2}\left(\int_{0}^{t} \int_{-\infty}^{\infty}\left(G_{x}^{2}+G_{\tau}^{2}\right) \theta^{2} d x d \tau\right)^{1 / 2} \\
& +c(\sup |G|)\left(\int_{-\infty}^{\infty} \theta^{2}(0) d x\right)^{1 / 2}\left(\int_{-\infty}^{\infty} \psi_{t}^{2}(0) d x\right)^{1 / 2} \\
& +c(\sup |G|)\left(\int_{-\infty}^{\infty} \theta^{2}(t) d x\right)^{1 / 2}\left(\int_{-\infty}^{\infty} \psi_{t}^{2}(t) d x\right)^{1 / 2} \\
& +c(\sup |\bar{G}|)\left(\int_{0}^{t}\left(\psi_{x}^{2}+\psi_{\tau}^{2}\right)(s \tau, \tau) d \tau\right) 1 / 2\left(\int_{0}^{t} \theta^{2}(s \tau, \tau) d \tau\right)^{1 / 2} \\
& \leqq c(\sup |G|)\left(N_{2}(t)^{2}+N_{2}(t) N_{3}(t)+N_{1}(t)^{2}\right) \\
& +c N_{2}(t)\left(\int_{0}^{t} \int_{-\infty}^{\infty}\left(G_{x}^{2}+G_{\tau}^{2}\right) \theta^{2} d x d \tau\right)^{1 / 2} \\
& +c(\sup |G|)\left(\delta+N_{2}(t)+N_{1}(t) N_{2}(t)\right)\left(\int_{0}^{t} \theta^{2}(s \tau, \tau) d \tau\right)^{1 / 2} .
\end{aligned}
$$

Combine (5.20), (5.23) (5.25), (5.28) to obtain

Lemma 5.2. Let $g$ be non-negative and define $G$ by (5.21). Then

$$
\begin{aligned}
& \int_{0}^{t} \int g \theta_{i}^{2} d x d \tau \leqq c(\sup |G|)\left(\delta N_{1}(t)+N_{1}(t)^{2}+N_{1}(t) N_{2}(t)^{2}+N_{2}(t)^{2}+N_{3}(t)^{2}\right) \\
& \quad+c N_{2}(t)\left(\int_{0}^{t} \int_{-\infty}^{\infty}\left(\left(\tilde{\lambda}_{i} G\right)^{2}+G_{x}^{2}+G_{\tau}^{2}\right) \theta^{2} d x d \tau\right)^{1 / 2} \\
& \quad+c(\sup |G|)\left(\delta+N_{2}(t)+N_{1}(t) N_{2}(t)\right)\left(\int_{0}^{t} \theta^{2}(s \tau, \tau) d \tau\right)^{1 / 2} .
\end{aligned}
$$

Since $N_{1}, N_{2}$ are bounded through Lemma 4.4 and we are in the process of bounding $N_{3}$, it will suffice to show that $(\sup |G|)<\delta$ and to bound the two integrals on the right of (5.29) for each case.

The estimate from the characteristic energy method is 
Lemma 5.3. For $\delta$ sufficiently small,

$$
\begin{aligned}
& \iint_{\Omega_{1}(t)}\left|\lambda_{1 x}\right| \theta_{2}^{2} d x d \tau+\iint_{\Omega_{2}(t)}\left|\lambda_{2 x}\right| \theta_{1}^{2} d x d \tau+\iint_{\Omega_{+}(t)}\left|\lambda_{1 x}\right| \theta_{1}^{2} d x d \tau \\
& \leqq c \delta\left(\delta N_{1}(t)+N_{1}(t)^{2}+N_{1}(t) N_{2}(t)^{2}+N_{2}(t)^{2}+\delta^{-1 / 2} N_{3}(t)^{2}\right) \\
& +c \delta\left(\delta+N_{2}(t)+N_{1}(t) N_{2}(t)\right)\left(\int_{0}^{t} \theta^{2}(s \tau, \tau) d \tau\right)^{1 / 2} .
\end{aligned}
$$

Proof. i) Estimate on $\left|\lambda_{1 x}\right| \theta_{2}^{2}$. Let $g=\chi_{1}\left|\lambda_{1 x}\right|$ in which $\chi_{1}=1$ for $x<0, \chi_{1}=0$ for $x>0$. Then

$$
g(x, \tau)= \begin{cases}\lambda_{1 x}(x, \tau)+O\left(\delta e^{-\gamma(|x|+\tau)}\right), & (x, \tau) \in \Omega_{+}, \\ -\lambda_{1 x}(x, \tau)+O\left(\delta e^{-\gamma(|x|+\tau)}\right), & (x, \tau) \in \Omega_{-}, \\ 0, & (x, \tau) \in \Omega_{2} .\end{cases}
$$

The relevant characteristics in (3.52) are $X_{2}$ which are entirely contained within $\Omega_{1}=\Omega_{+} \cup \Omega_{-}$(cf. Fig. 2) and in $\Omega_{1}$ the characteristics are straight lines so that $\partial X_{2} / \partial x \equiv 1$. Fix $(x, \tau) \in \Omega_{1}$ and denote

$$
\begin{aligned}
& {\left[T_{1}, T_{2}\right]=\left\{\sigma: \tau<\sigma,\left(X_{2}(\sigma, \tau, x), \sigma\right) \in \Omega_{+}\right\},} \\
& {\left[T_{3}, T_{4}\right]=\left\{\sigma: \tau<\sigma,\left(X_{2}(\sigma, \tau, x), \sigma\right) \in \Omega_{-}\right\} .}
\end{aligned}
$$

Depending on the sign of $\kappa$ and the location of $(x, \tau)$, the $T_{i}$ 's take on the values $\tau, \infty$ (endpoints), $\tau-x / \lambda_{2}^{-}$(intersection with $x=0$ ), or $T$ satisfying

$$
y_{1}=y\left(X_{2}, T\right)=\left(X_{2}(T, \tau, x)-\lambda_{1}^{-} T\right) /(\sqrt{\alpha T})=\left(x-\lambda_{2}^{-} \tau+\left(\lambda_{2}^{-}-\lambda_{1}^{-}\right) T\right) /(\sqrt{\alpha T})
$$

[intersection with $\left(\Omega_{+}, \Omega_{-}\right.$border $\left.)\right]$. It follows that for $(x, \tau)$ large, $T=O(x)+O(\tau)$, and thus that for each $i$

$$
\left|\partial_{t} T_{i}\right|+\left|\partial_{x} T_{i}\right|<c,
$$

with $c$ a constant independent of $x, \tau, \delta$.

In $\Omega_{1},(\partial / \partial \sigma) \lambda_{1}\left(X_{2}(\sigma, \tau, x), \sigma\right)=\lambda_{2}^{-} \lambda_{1 x}+\lambda_{1 \sigma}$ and $\left|\lambda_{1 \sigma}\right|<c \delta(1+\sigma)^{-3 / 2}$. Thus

$$
\int_{T_{1}}^{T_{2}}\left|\lambda_{1 \sigma}\right| d \sigma+\int_{T_{3}}^{T_{4}}\left|\lambda_{1 \sigma}\right| d \sigma \leqq \int_{\tau}^{\infty} c \delta(1+\sigma)^{-3 / 2} d \sigma \leqq c \delta(1+\tau)^{-1 / 2} .
$$

Using $\partial X_{2} / \partial x=1,(5.35)$ and (5.31), we may write

$$
\begin{aligned}
G(x, \tau) & =\int_{T_{1}}^{T_{2}} \lambda_{1 x}\left(X_{2}(\sigma, \tau, x), \sigma\right) d \sigma-\int_{T_{3}}^{T_{4}} \lambda_{1 x}\left(X_{2}(\sigma, \tau, x), \sigma\right) d \sigma \\
& =\int_{T_{1}}^{T_{2}}\left(\lambda_{1 x}+\left(\lambda_{2}^{-}\right)^{-1} \lambda_{1 \sigma}\right) d \sigma-\int_{T_{3}}^{T_{4}}\left(\lambda_{1 x}+\left(\lambda_{2}^{-}\right)^{-1} \lambda_{1 \sigma}\right) d \sigma+O\left(\delta(1+\tau)^{-1 / 2}\right) \\
& \left.\left.=\left(\lambda_{2}^{-}\right)^{-1}\left(\lambda_{1}\right]_{T_{1}}^{T_{2}}-\lambda_{1}\right]_{T_{3}}^{T_{4}}\right)+O\left(\delta(1+\tau)^{-1 / 2}\right) \leqq c \delta(1+\tau)^{-1 / 2}
\end{aligned}
$$

since (2.31) implies that $\lambda_{1}=\lambda_{1}^{-}+O(1+\tau)^{-1 / 2}$ for $x<0$. Next differentiate $G$ to obtain

$$
\begin{aligned}
\left|G_{x}\right|= & \left(\lambda_{2}^{-}\right)^{-1} \sum_{i=1}^{4} \beta_{i}\left\{\left(\partial_{x} T_{i}\right) \lambda_{2}^{-} \lambda_{1 x}\left(X_{2}\left(T_{i}, \tau, x\right), T_{i}\right)\right. \\
& \left.+\lambda_{1 \sigma}\left(X_{2}\left(T_{i}, \tau, x\right), T_{i}\right)+\lambda_{1 x}\left(X_{2}\left(T_{i}, \tau, x\right), T_{i}\right)\right\}+O\left(\delta(1+\tau)^{-1}\right) \leqq c \delta(1+\tau)^{-1}
\end{aligned}
$$


in which $\beta_{i}= \pm 1$. Similarly,

$$
\left|G_{\tau}\right| \leqq c \delta(1+\tau)^{-3 / 2}
$$

Moreover $G \equiv 0$ for $x>0$ and $\left|\tilde{\lambda}_{i}\right|<c \delta(1+\tau)^{-1 / 2}$ for $x<0$. Therefore

$$
\begin{gathered}
\sup |G|<c \delta \\
\int_{0}^{t} \int_{-\infty}^{\infty}\left(\left(\tilde{\lambda}_{i} G\right)^{2}+G_{x}^{2}+G_{\tau}^{2}\right) \theta^{2} d x d \tau \leqq c \delta^{2} \int_{0}^{t} \int_{-\infty}^{\infty}(1+\tau)^{-2} \theta^{2} d x d \tau \leqq c \delta^{2} N_{1}(t)^{2}
\end{gathered}
$$

Using (5.39) in (5.29) establishes the bound (5.30) for the integral of $\left|\lambda_{1 x}\right| \theta_{2}^{2}$.

ii) Estimate of $\left|\lambda_{1 x}\right| \theta_{1}^{2}$. Let $g=\chi_{1}\left|\lambda_{1 x}\right|$ as in (i). Now the relevant characteristics are $X_{1}(\sigma, \tau, x)$ with $X_{1}<0$ which may enter $\Omega_{1}$ from $\Omega_{2}$ and may cross $X_{1}=s \sigma$ as well as $y=y_{1}$. Thus $\left(\partial X_{1} / \partial x\right)(\sigma, \tau, x)$ has values 1 for $x<s \tau$ and $\left(s-\lambda_{1}^{-}\right) /\left(s-\lambda_{1}^{+}\right)$for $x>s \tau$. Denote

$$
\begin{aligned}
& {\left[T_{1}, T_{2}\right]=\left\{\sigma: \tau \leqq \sigma,\left(X_{1}(\sigma, \tau, x), \sigma\right) \in \Omega_{+}\right\},} \\
& {\left[T_{3}, T_{4}\right]=\left\{\sigma: \tau \leqq \sigma,\left(X_{1}(\sigma, \tau, x), \sigma\right) \in \Omega_{-}\right\} .}
\end{aligned}
$$

Depending on the sign of $\kappa$ and the position of $(x, \tau)$, each $T_{i}$ takes on one of the values $s_{1}=\tau, \quad s_{2}=\infty \quad$ (lower and upper limits), $s_{3}=\left(x-\lambda_{1}^{-} \tau\right) / \lambda_{1}^{-}$, $s_{4}=-\left(s-\lambda_{1}^{-}\right)\left(x-\lambda_{1}^{+} \tau\right) / \lambda_{1}^{-}\left(s-\lambda_{1}^{+}\right)$(intersections with $X_{1}=0$ for $0<x<s \tau$ and $s \tau<x$ respectively), or $s_{5}$ satisfying $y=y_{1}$, i.e.

$$
y_{1}=\frac{X_{1}\left(s_{5}, \tau, x\right)-\lambda_{1}^{-} s_{5}}{\sqrt{\alpha s_{5}}} .
$$

There are three possibilities depending on whether $x<0,0<x<s \tau$ or $x>s \tau$; however in each case $X_{1}\left(s_{5}, \tau, x\right)=d_{1} x+d_{2} \tau+\lambda_{1}^{-} s_{5}$ (with three different positive values of $d_{1}, d_{2}$ ). Thus

$$
\begin{gathered}
s_{5}=\alpha y_{1}^{-2}\left(d_{1} x+d_{2} \tau\right)^{2}, \quad \partial_{x} s_{5}=2 d_{1} \alpha y_{1}^{-2}\left(d_{1} x+d_{2} \tau\right), \quad \partial_{\tau} s_{5}=2 d_{2} \alpha y_{1}^{-2}\left(d_{1} x+d_{2} \tau\right), \\
\left|\lambda_{1 x}\left(X_{1}\left(s_{5}, \tau, x\right), s_{5}\right)\right| \leqq c \delta\left(1+s_{5}\right)^{-1} .
\end{gathered}
$$

For $i<5,\left|\partial_{x} s_{i}\right|+\left|\partial_{\tau} s_{i}\right|<c$ and $\left|\lambda_{1 x}\left(\sigma=s_{i}\right)\right| \leqq c \delta(1+\tau)^{-1}$. Thus for each $i \leqq 5$,

$$
\left(\left|\partial_{x} s_{i}\right|+\left|\partial_{\tau} s_{i}\right|\right)\left|\lambda_{1 x}\right|\left(X_{1}\left(s_{i}, \tau, x\right), s_{i}\right) \leqq c \delta(1+\tau)^{-1} .
$$

Evaluate $G$ as

$$
G(x, \tau)=\kappa_{1} \int_{T_{1}}^{T_{2}} \lambda_{1 x} d \sigma-\kappa_{2} \int_{T_{3}}^{T_{4}} \lambda_{1 x} d \sigma+O\left(e^{-\gamma \tau}\right)
$$

in which $\kappa_{1}, \kappa_{2}$ are piecewise constant, having a jump along $x=s \tau$ and taking on values either 1 or $\left(s-\lambda_{1}^{-}\right) /\left(s-\lambda_{1}^{+}\right)$. As before $\lambda_{1 x}$ may be replaced by $(\partial / \partial \sigma) \lambda_{1}$ by adding in terms proportional to $\lambda_{1 \sigma}$ so that $G=G(x, \tau)$ satisfies

$$
\begin{aligned}
G & \left.\left.=\left(\lambda_{2}^{-}\right)^{-1}\left(\kappa_{1} \lambda_{1}\right]_{T_{1}}^{T_{2}}-\kappa_{2} \lambda_{1}\right]_{T_{3}}^{T_{4}}\right)+O\left(\delta(1+\tau)^{-1 / 2}\right) \leqq c \delta(1+\tau)^{-1 / 2}, \\
\left|G_{x}\right| & =\left|\left(\lambda_{2}^{-}\right)^{-1} \sum_{i=1}^{4} \beta_{i}\left\{\left(\partial_{x} T_{i}\right)\left(\lambda_{2}^{-} \lambda_{1 x}+\lambda_{1 \tau}\right)+\lambda_{1 x} \partial_{x} X_{1}\right\}+O\left(\delta(1+\tau)^{-1}\right)\right| \\
& \leqq c \delta(1+\tau)^{-1} \\
\left|G_{\tau}\right| & \leqq c \delta(1+\tau)^{-1}
\end{aligned}
$$


in which the $\delta$-function part of $G_{x}, G_{t}$ is ignored as in (5.26). That part is compensated for by the integral along $x^{\prime}=s t^{\prime}$ in (5.30). Also for all $x$,

Thus

$$
\left|\tilde{\lambda}_{1}\right| \leqq c \delta(1+\tau)^{-1 / 2}+c\left|\lambda_{2 x}\right|
$$

$$
\begin{gathered}
\sup |G|<\delta, \\
\int_{0}^{t} \int\left(\left(\tilde{\lambda}_{1} G\right)^{2}+G_{x}^{2}+G_{\tau}^{2}\right) \theta^{2} d x d \tau \leqq c \delta^{2} \int_{0}^{t} \int\left((1+\tau)^{-2}+\delta\left|\hat{\lambda}_{2 x}\right|\right) \theta^{2} d x d \tau \\
\leqq c \delta^{2} N_{1}(t)^{2}+c \delta^{3} N_{3}(t)^{2} .
\end{gathered}
$$

Substitute (5.45) into (5.29) to complete the estimate for $\left|\lambda_{1 x}\right| \theta_{1}^{2}$ in (5.30).

iii) Estimate of $\left|\lambda_{2 x}\right| \theta_{1}^{2}$. Let $g=\chi_{2}\left|\lambda_{2 x}\right|$ with $\chi_{2}$ the characteristic function of $\Omega_{2}(t)$, i.e.

$$
\begin{aligned}
g(x, \sigma) & = \begin{cases}\left|\lambda_{2 x}(x, \sigma)\right|, & x>0 \\
0, & x<0\end{cases} \\
& = \begin{cases}-\lambda_{2 x}\left(f_{1}(x-s \sigma)\right)+O\left(\delta e^{-\gamma(|x|+\sigma)}\right), & x>0 \\
0, & x<0\end{cases}
\end{aligned}
$$

The relevant characteristics here are $X_{1}(\sigma, \tau, x)$ with $X_{1}>0$ (i.e. $X_{1} \in \Omega_{2}$ ). In $\Omega_{2}$ the $X_{1}$ characteristic has piecewise constant slope, so that for $\sigma>\tau$,

$$
\frac{\partial X_{1}}{\partial x}(\sigma, \tau, x)=\left\{\begin{array}{lll}
1 & \text { if } & \left(x>s \tau, X_{1}>s \sigma\right) \\
\left(s-\lambda_{1}^{-}\right) /\left(s-\lambda_{1}^{+}\right) & \text {if } \quad x>s \tau, X_{1}<s \sigma
\end{array} \quad \text { or } \quad\left(x<s \tau, X_{1}<s \sigma\right) .\right.
$$

Denote

$$
\begin{aligned}
& {\left[T_{1}, T_{2}\right]=\left\{\sigma: \tau<\sigma, X_{1}(\sigma, \tau, x)>s \sigma\right\},} \\
& {\left[T_{2}, T_{3}\right]=\left\{\sigma: \tau<\sigma, 0<X_{1}(\sigma, \tau, x)<s \sigma\right\} .}
\end{aligned}
$$

If $x<s \tau$ so that $X_{1}<s \sigma$ for all $\sigma>\tau$, set $T_{1}=T_{2}=\tau$. We can identify $T_{i}$ as $T_{1}=\tau$,

$$
\begin{aligned}
& T_{2}= \begin{cases}\tau, & \text { if } x<s \tau \\
\left(x-\lambda_{1}^{+} \tau\right) /\left(s-\lambda_{1}^{+}\right), & \text {if } x>s \tau\end{cases} \\
& T_{3}= \begin{cases}\tau-x / \lambda_{1}^{-}, & \text {if } x<s \tau . \\
-\left(x-\lambda_{1}^{+} \tau\right)\left(s-\lambda_{1}^{-}\right) /\left(s-\lambda_{1}^{+}\right) \lambda_{1}^{-}, x>s \tau & x>s \tau .\end{cases}
\end{aligned}
$$

As before for each $i,\left|\partial_{t} T_{i}\right|+\left|\partial_{x} T_{i}\right|<c$. Note that $X_{1}\left(T_{3}, \tau, x\right)=0$. Now evaluate $G$ as

$$
\begin{aligned}
G(x, \tau)= & -\left(s-\lambda_{1}^{+}\right)\left(s-\lambda_{1}^{-}\right)^{-1} \int_{\tau}^{T_{2}} \lambda_{2 x}\left(x+\lambda_{1}^{+}(\sigma-\tau)-s \sigma\right) d \sigma \\
& -\int_{T_{2}}^{T_{3}} \lambda_{2 x}\left(X_{1}\left(T_{2}, \tau, x\right)+\lambda_{1}^{-}\left(\sigma-T_{2}\right)-s \sigma\right) d \sigma+O\left(\delta e^{-\gamma \tau}\right) \\
= & \left(s-\lambda_{1}^{-}\right)^{-1}\left\{\int_{\tau}^{T_{2}}(\partial / \partial \sigma) \lambda_{2} d \sigma+\int_{T_{2}}^{T_{3}}(\partial / \partial \sigma) \lambda_{2} d \sigma\right\}+O\left(\delta e^{-\gamma \tau}\right) \\
= & \left(s-\lambda_{1}^{-}\right)^{-1}\left\{\lambda_{2}\left(0, T_{3}\right)-\lambda_{2}(x, \tau)\right\}+O\left(\delta e^{-\gamma \tau}\right) \leqq c \delta,
\end{aligned}
$$

since $\lambda_{2}$ has total variation of size $\delta$. Next

$$
\begin{aligned}
\left|G_{x}(x, \tau)\right| & =\left(s-\lambda_{1}^{-}\right)^{-1}\left\{X_{1 x}\left(T_{3}, \tau, x\right) \lambda_{2 x}\left(0, T_{3}\right)-\lambda_{2 x}(x, \tau)\right\}+O\left(\delta e^{-\gamma \tau}\right) \\
& \leqq c\left(\left|\lambda_{2 x}(x, \tau)\right|+\delta e^{-\gamma \tau}\right),
\end{aligned}
$$


since $\left|\lambda_{2 x}\left(0, T_{3}\right)\right| \leqq c \delta e^{-\gamma T_{3}} \leqq c \delta e^{-\gamma \tau}$. Similarly

$$
\left|G_{\tau}(x, \tau)\right| \leqq c\left(\left|\lambda_{2 x}(x, \tau)\right|+\delta e^{-\gamma \tau}\right)
$$

Also in $x>0,\left|\tilde{\lambda}_{2}\right| \leqq c\left|\lambda_{2 x}\right| \leqq c \delta$. Thus

$$
\begin{gathered}
\sup |G|<c \delta, \\
\int_{0}^{t} \int\left(\left(\tilde{\lambda}_{1} G\right)^{2}+G_{x}^{2}+G_{\tau}^{2}\right) \theta^{2} d x d \tau \\
\leqq c \int_{0}^{t} \int\left(\delta\left|\lambda_{2 x}\right|+\delta^{2} e^{-2 \gamma \tau}\right) \theta^{2} d x d \tau \\
\leqq c \delta^{2} N_{1}(t)^{2}+c \delta N_{3}(t)^{2} .
\end{gathered}
$$

Substitute (5.53) into (5.29) to complete the estimate for $\left|\lambda_{2 x}\right| \theta_{1}^{2}$ in (5.30). This completes the proof of Lemma 5.3.

Note. The same estimation method does not work for $\iint_{\Omega_{2}(t)}\left|\lambda_{2 x}\right| \theta_{2}^{2} d x d \tau$, because the $X_{2}$ characteristics have endpoints on the line $x^{\prime}=s t^{\prime}$ on which $\left|\lambda_{2 x}\right|$ is large.

Fortunately this term was estimated in Lemma 5.1 .
We finish the characteristic energy estimates by estimating $\int_{0}^{t} \theta^{2}(s \tau, \tau) d \tau$. For $x \geqq s t$,

$$
\begin{aligned}
\theta_{i}(x, t)^{2}= & \frac{1}{2} \int_{0}^{t} \theta_{i}\left\{\sum_{k=1}^{2} \theta_{k}\left(l_{i \tau}+\lambda_{i} l_{i x}\right) \cdot r_{k}\right. \\
& \left.+B^{-1}\left(\psi_{x x}-\psi_{\tau \tau}-\Gamma-K-H\right)-\tilde{\lambda}_{i} \theta_{i x}\right\}\left(x-\bar{\lambda}_{i}^{+}(t-\tau), \tau\right) d \tau+\theta_{i}\left(x-\bar{\lambda}_{i}^{+} t, 0\right)^{2} .
\end{aligned}
$$

Thus

$$
\begin{aligned}
\int_{0}^{t} \theta_{i}(s \tau, \tau)^{2} d \tau= & s^{-1} \int_{0}^{s t} \theta_{i}\left(x, s^{-1} x\right)^{2} d x \leqq s^{-1} \int_{0}^{s t} \theta_{i}\left(x, s^{-1} x\right)^{2} d x+\int_{s t}^{\infty} \theta_{i}(x, t)^{2} d x \\
= & \frac{1}{2}\left(s-\bar{\lambda}_{i}^{+}\right)^{-1} \int_{0}^{t} \int_{s \tau}^{\infty} \theta_{i}\left\{\sum_{k=1}^{2} \theta_{k}\left(l_{i \tau}+\lambda_{i} l_{i x}\right) \cdot r_{k}\right. \\
& \left.+B^{-1}\left(\psi_{x x}-\psi_{\tau \tau}-\Gamma-K-H\right)-\tilde{\lambda}_{i} \theta_{i x}\right\}(x, \tau) d x d \tau+\int_{0}^{\infty} \theta_{i}(x, 0)^{2} d x
\end{aligned}
$$

Estimate various terms separately as

$$
\begin{aligned}
&\left|\int_{0}^{t} \int_{s \tau}^{\infty} \theta_{i} \sum_{k=1}^{2} \theta_{k}\left(l_{i \tau}+\lambda_{i} l_{i x}\right) \cdot r_{k} d x d \tau\right| \leqq \int_{0}^{t} \int_{-\infty}^{\infty} \theta^{2}\left(\left|\lambda_{1 x}\right|+\left|\lambda_{2 x}\right|\right) d x d \tau \leqq N_{3}(t)^{2}, \\
& \int_{0}^{\infty} \theta_{i}^{2}(x, 0) d x \leqq N_{1}(t)^{2}, \\
&\left|\int_{0}^{t} \int_{s \tau}^{\infty} \theta_{i} B^{-1}(\Gamma+K+H) d x d \tau\right| \leqq c N_{1}(t)\left(\delta+\delta N_{2}(t)+N_{2}(t)^{2}\right),
\end{aligned}
$$

by Lemma 4.2. Next since $\left|\tilde{\lambda}_{i}\right| \leqq c\left|\lambda_{2 x}\right|<c \delta$ for $x>0$,

$$
\begin{aligned}
\left|\int_{0}^{t} \int_{s \tau}^{\infty} \tilde{\lambda}_{i} \theta_{i} \theta_{i x} d x d \tau\right| & \leqq\left(\int_{0}^{t} \int_{0}^{\infty} \theta_{i x}^{2} d x d \tau\right)^{1 / 2}\left(\int_{0}^{t} \int_{0}^{\infty} \tilde{\lambda}_{i}^{2} \theta_{i}^{2} d x d \tau\right)^{1 / 2} \\
& \leqq c \delta^{1 / 2} N_{2}(t) N_{3}(t)
\end{aligned}
$$


Finally, using (5.27) in the last step,

$$
\begin{aligned}
&\left|\int_{0}^{t} \int_{s \tau}^{\infty} B^{-1} \theta_{i}\left(\psi_{x x}-\psi_{\tau \tau}\right) d x d \tau\right|=\mid \int_{0}^{t} \int_{s \tau}^{\infty} B^{-1}\left(-\theta_{i x} \psi_{x}+\theta_{i \tau} \psi_{\tau}\right)-\left(B^{-1}\right)_{x} \theta_{i} \psi_{x} \\
&+\left(B^{-1}\right)_{\tau} \theta_{i} \psi_{\tau} d x d \tau+\int_{0}^{\infty} B^{-1} \theta_{i} \psi_{\tau}(x, 0) d x-\int_{0}^{s t} B^{-1} \theta_{i} \psi_{\tau}\left(x, s^{-1} x\right) d x \\
& \quad-\int_{s t}^{\infty} B^{-1} \theta_{i} \psi_{\tau}(x, t) d x-\int_{0}^{t} B^{-1} \theta_{i} \psi_{x}(s \tau, \tau) d \tau \mid \\
& \leqq {\left[N_{2}(t)^{2}+\delta^{1 / 2} N_{2}(t) N_{3}(t)+N_{1}(t)^{2}\right.} \\
&-\left.\left(\int_{0}^{t}\left(\psi_{x}^{2}+\psi_{\tau}^{2}\right)(s \tau, \tau) d \tau\right)^{1 / 2}\left(\int_{0}^{t} \theta^{2}(s \tau, \tau) d \tau\right)^{1 / 2}\right] \\
& \leqq {\left[N_{1}(t)^{2}+N_{2}(t)^{2}+\delta^{1 / 2} N_{2}(t) N_{3}(t)\right.} \\
&+\left.\left(\delta+N_{2}(t)+N_{1}(t) N_{2}(t)\right)\left(\int_{0}^{t} \theta^{2}(s \tau, \tau) d \tau\right)^{1 / 2}\right] .
\end{aligned}
$$

Combine (5.55)-(5.59) to obtain

$$
\begin{aligned}
\int_{0}^{t} \theta^{2}(s \tau, \tau) d \tau \leqq & c\left[\delta N_{1}(t)+N_{1}(t)^{2}+N_{2}(t)^{2}+N_{1}(t) N_{2}(t)^{2}+N_{3}(t)^{2}\right] \\
& +c\left(\delta+N_{1}(t) N_{2}(t)+N_{2}(t)\right)\left(\int_{0}^{t} \theta^{2}(s \tau, \tau) d \tau\right)^{1 / 2},
\end{aligned}
$$

from which it follows that

Lemma 5.4. For $\delta$ sufficiently small,

$$
\int_{0}^{t} \theta^{2}(s \tau, \tau) d \tau \leqq c\left[\delta^{2}+N_{1}^{2}+N_{1}^{2} N_{2}^{2}+N_{2}^{2}+N_{3}^{2}\right](t) .
$$

Now combine (5.30) of Lemma 5.3 with (5.61) of Lemma 5.4 to obtain, after some recombination.

$$
\begin{gathered}
\iint_{\Omega_{1}(t)}\left|\lambda_{1 x}\right| \theta_{2}^{2} d x d \tau+\iint_{\Omega_{2}(t)}\left|\lambda_{2 x}\right| \theta_{1}^{2} d x d \tau+\iint_{\Omega_{+}(t)}\left|\lambda_{1 x}\right| \theta_{1}^{2} d x d \tau \\
\leqq c \delta\left(\delta^{2}+N_{1}^{2}+N_{1}^{2} N_{2}^{2}+N_{2}^{2}+\dot{\delta}^{-1 / 2} N_{3}^{2}\right)(t)
\end{gathered}
$$

Combine this with (4.27) of Lemma 4.4 to obtain

$$
N_{1}\left(N_{1}-c \delta-c \delta N_{2}-c N_{2}^{2}-c \delta N_{1} N_{2}^{2}\right)+N_{2}^{2} \leqq c N_{1}(0)^{2}+c \delta^{3}+c \delta^{1 / 2} N_{3}^{2} .
$$

Next combine (5.62) with (5.6) of Lemma 5.1 using the inequality

$$
N_{3}(t)^{2} \leqq c \iint_{\Omega_{1}(t)}\left|\lambda_{1 x}\right| \theta^{2} d x d \tau+c \iint_{\Omega_{2}(t)}\left|\lambda_{2 x}\right| \theta^{2} d x d \tau
$$

to obtain

$$
\left.N_{3}^{2} \leqq c N_{1}\left\{\delta+N_{1}+\delta N_{1} N_{2}^{2}+\delta N_{2}+N_{2}^{2}\right)\right\}+c N_{2}^{2}+c \delta^{3}
$$


Finally combine (5.64) with (5.63) to obtain

$$
N_{1}\left(N_{1}-c \delta-c \delta N_{2}-c N_{2}^{2}-c \delta N_{1} N_{2}^{2}\right)+N_{2}^{2} \leqq c N_{1}(0)^{2}+c \delta^{3} .
$$

This inequality implies that either $N_{1}$ and $N_{2}$ are both small or that $N_{1}, N_{2}$ are of $O(1)$ size independent of $\delta$. However since $N_{1}, N_{2}$ start off with size $\delta^{2}$ and are continuous, they must remain small. It follows that, for $N_{1}(0)$ sufficiently small,

$$
N_{1}^{2}+N_{2}^{2}+N_{3}^{2} \leqq c \delta^{2}+c N_{1}(0)^{2} .
$$

By assumption $\|\phi, \psi\|_{2}(0)^{2}+\left\|\partial_{t} \psi\right\|_{1}(0)^{2} \leqq c \delta^{2}$. This establishes the main result of this paper.

Theorem 5.1. Let $\delta$ be sufficiently small, then for any $t \geqq 0$,

$$
\sup _{0 \leqq \tau \leqq t}\left\{\|\phi, \psi\|_{2}(\tau)^{2}+\left\|\partial_{t} \psi\right\|_{1}(\tau)^{2}\right\}+\int_{0}^{t}\left\|\phi_{x}, \psi_{x}, \psi_{t}\right\|_{1}^{2} d \tau \leqq c \delta^{2} .
$$

Finally we prove Theorem 1. Since $\phi_{x}=\varrho_{*}, \psi_{x}=m_{*}, \psi_{t}=-z_{*}$, then

$$
\sup _{0 \leqq \tau \leqq t}\left\|\varrho_{*}, m_{*}, z_{*}\right\|_{1}(\tau)^{2}+\int_{0}^{t}\left\|\varrho_{*}, m_{*}, z_{*}\right\|_{1}^{2} d \tau \leqq c \delta^{2} .
$$

Since this inequality is also satisfied by $\left(\varrho_{2}, m_{2}, z_{2}\right)$ and $\left(\varrho_{3}, m_{3}, z_{3}\right)$, we obtain

$$
\sup _{0 \leqq \tau \leqq t}\left\|\left(\varrho-\varrho_{1}\right),\left(m-m_{1}\right),\left(z-z_{1}\right)\right\|_{1}(\tau)^{2}+\int_{0}^{t}\left\|\left(\varrho-\varrho_{1}\right),\left(m-m_{1}\right),\left(z-z_{1}\right)\right\|_{1}^{2} d \tau \leqq c \delta^{2} .
$$

Use the formula for $f$ in terms of $\varrho, m, z$ and the equation for $f_{t}$ in terms of $f_{x}$ and $f$ to obtain

$$
\begin{aligned}
\sup _{0 \leqq \tau \leqq t} \int_{-\infty}^{\infty}\left(f-f_{1}\right)^{2} & +\left(f_{x}-f_{1 x}\right)^{2}+\left(f_{t}-f_{1 t}\right)^{2} d x \\
& +\int_{0}^{t} \int_{-\infty}^{\infty}\left(f-f_{1}\right)^{2}+\left(f_{x}-f_{1 x}\right)^{2}+\left(f_{t}-f_{1 t}\right)^{2} d x d \tau \leqq c \delta^{2}
\end{aligned}
$$

which is the inequality (1.8).

We now prove (1.9). From (4.25), (4.26) and integration of $\psi_{t}$ times (3.18), we have

$$
\begin{aligned}
\left\|\psi_{t}, \phi_{x}, \psi_{x}\right\|_{1}^{2}\left(t_{2}\right) \leqq c\left\|\psi_{t}, \phi_{x}, \psi_{x}\right\|_{1}^{2}\left(t_{1}\right)+c \int_{t_{1}}^{t_{2}}\left\|\phi_{x}, \psi_{x}, \psi_{t}\right\|_{1}^{2} d \tau \\
+c \int_{t_{1}}^{t_{2}} \int_{-\infty}^{\infty}\left|\lambda_{1 x}\right| \psi^{2} d x d \tau, \quad 0 \leqq t_{1} \leqq t_{2}<\infty .
\end{aligned}
$$

This implies (by integration of $t_{1}$ ) that

$$
\left\|\phi_{x x}, \psi_{x x}\right\|^{2}(t) \leqq c \int_{t}^{t}\left\|\phi_{x}, \psi_{x}, \psi_{t}\right\|_{1}^{2} d \tau+c \int_{t}^{t} \int_{-\infty}^{\infty}\left|\lambda_{1 x}\right| \psi^{2} d x d \tau
$$

The estimate (5.66) implies

$$
\int_{0}^{t}\left\|\phi_{x}, \psi_{x}, \psi_{t}\right\|_{1}^{2} d \tau+\int_{0}^{t} \int_{-\infty}^{\infty}\left|\lambda_{1 x}\right| \psi^{2} d x d \tau \leqq c \delta^{2}
$$

which is slightly stronger than (5.67). It follows that the right-hand side of (5.71) tends to zero as $t \rightarrow \infty$. This proves (1.9) and concludes the proof of Theorem 1. 


\section{Appendix A. The Chapman-Enskog Error for the Broadwell Equation}

Suppose that $\varrho, m$ and $z=z(\varrho, m)$ satisfy the model Navier-Stokes equations with errors, i.e.

$$
\varrho_{t}+m_{x}=e_{1}, \quad m_{t}+z(\varrho, m)_{x}=e_{2},
$$

with $u=m / \varrho$ and

$$
z=\varrho F(u)-v(u) u_{x},
$$

in which $F$ and $v$ are given by (2.11), (2.16). The third Broadwell equation $\left(z_{t}+m_{x}=Q(f, f)\right)$ then has the error

$$
E=Q(f, f)-\left(z_{t}+m_{x}\right)=\frac{1}{8}\left\{(\varrho-z)^{2}-4\left(z^{2}-m^{2}\right)\right\}-\left(z_{t}+m_{x}\right) .
$$

To calculate $E$, first eliminate $z=\varrho F-v u_{x}$ to get

$$
E=\frac{1}{8}\left\{\varrho^{2}-2 \varrho^{2} F-3 \varrho^{2} F^{2}+4 m^{2}\right\}+\left\{\frac{1}{4}(\varrho+3 \varrho F) v u_{x}-\frac{3}{8}\left(v u_{x}\right)^{2}-\left(\varrho F-v u_{x}\right)_{t}-m_{x}\right\} .
$$

The undifferentiated terms vanish due to the definition of $F$. Next eliminate time derivatives $(\varrho F)_{t}$ using the Navier-Stokes equations to obtain

$$
\begin{aligned}
E= & \left\{\frac{1}{4} \varrho(1+3 F) v u_{x}+\left(F-u F^{\prime}-1\right) m_{x}+F^{\prime}(\varrho F)_{x}\right\} \\
& +\left\{-F^{\prime}\left(v u_{x}\right)_{x}+\left(v u_{x}\right)_{t}-\frac{3}{8}\left(v u_{x}\right)^{2}\right\}+\left\{\left(-F+u F^{\prime}\right) e_{1}-F^{\prime} e_{2}\right\} .
\end{aligned}
$$

The terms in the first bracket, which are linear in first derivatives, vanish due to the choice of viscosity $v$. Therefore

$$
E=\left\{-F^{\prime}\left(v u_{x}\right)_{x}+\left(v u_{x}\right)_{t}-\frac{3}{8}\left(v u_{x}\right)^{2}\right\}+\left(-F+u F^{\prime}\right) e_{1}-F^{\prime} e_{2} .
$$

\section{References}

1. Beale, J.T.: Large-time behavior of the Broadwell model of a discrete velocity gas. Commun. Math. Phys. 102, 217- 236 (1986)

2. Broadwell, J.E.: Shock structure in a simple discrete velocity gas. Phys. Fl. 7, 1243-1247 (1964)

3. Caflisch, R.E., Papanicolaou, G.C.: The fluid-dynamical limit of a nonlinear model Boltzmann equation. Commun. Pure Appl. Math. 32, 589-619 (1979)

4. Caflisch, R.E.: Navier-Stokes and Boltzmann shock profiles for a model of gas dynamics. Commun. Pure Appl. Math. 32, 521-554 (1979)

5. Goodman, J.: Nonlinear asymptotic stability of viscous shock profiles for conservation laws. Arch. Rat. Mech. Anal. 95, 325-344 (1986)

6. Kawashima, S., Matsumura, A.: Asymptotic stability of traveling wave solutions of systems for one-dimensional gas motion. Commun. Math. Phys. 101, 97-127 (1985)

7. Liu, T.-P.: Nonlinear stability of shock waves for viscous conservation laws. Mem. Am. Math. Soc. 56, 328 (1985)

8. Liu, T.-P.: Shock waves for compressible Navier-Stokes are stable. Commun. Pure Appl. Math. 39, 565-594 (1986)

9. Platkowski, T., Illner, R.: Discrete velocity models of the Boltzmann equation: a survey on the mathematical aspects of the theory. SIAM Rev. (to appear)

10. Tartar, L.: Existence globale pour un systeme hyperbolique semilinearie de la theorie cinetique des gaz. Semin. Goulaouic-Schwarz, No. $1(1975 / 76)$

Communicated by J. L. Lebowitz

Received April 21, 1987 